\title{
miR-503 suppresses the proliferation and metastasis of esophageal squamous cell carcinoma by triggering autophagy via PKA/mTOR signaling
}

\author{
JIAN WU ${ }^{1 *}$, FENGXIA GAO $^{2 *}$, TAO XU ${ }^{1}$, XIN DENG $^{3,4}$, CHAO WANG $^{1}$, XIAOYAN YANG $^{1}, \mathrm{ZHI} \mathrm{HU}^{1}$, \\ YANG LONG ${ }^{5}$, XUEMEI HE ${ }^{5}$, GUANNAN LIANG ${ }^{5}$, DELIAN REN ${ }^{2}$ and TIANYANG DAI ${ }^{1}$
}

${ }^{1}$ Department of Thoracic Surgery, The Affiliated Hospital of Southwest Medical University; ${ }^{2}$ Department of Immunology, College of Basic Medicine, Southwest Medical University; ${ }^{3}$ Drug Discovery Research Center, Southwest Medical University;

${ }^{4}$ Laboratory for Cardiovascular Pharmacology of the Department of Pharmacology, School of Pharmacy, Southwest Medical University; ${ }^{5}$ Experiment Medicine Center, The Affiliated Hospital of Southwest Medical University, Luzhou, Sichuan 646000, P.R. China

Received October 10, 2017; Accepted February 14, 2018

DOI: $10.3892 /$ ijo.2018.4320

\begin{abstract}
MicroRNA (miR)-503 is involved in the regulation of the malignant phenotype in multiple tumor types, and has been proven to be a novel diagnostic and therapeutic target; however, its function and mechanisms of action have not yet been fully elucidated in esophageal squamous cell carcinoma (ESCC). In the current study, we detected miR-503 expression by RT-qPCR and found that miR-503 expression was increased in ESCC, but negatively correlated with lymph node metastasis, TNM stage and tumor differentiation. Functionally, we confirmed that miR-503 inhibited the proliferation and metastasis of ESCC cells by triggering cellular autophagy. Mechanistically, we confirmed that miR-503 exerted its biological effects by targeting protein kinase CAMP-activated catalytic subunit alpha (PRKACA) in ESCC by dual luciferase reporter assay. Moreover, miR-503 was found to trigger autophagy in ESCC cells through the protein kinase A (PKA)/mammalian target of rapamycin (mTOR) pathway. Taken together, our results demonstrate that miR-503 suppresses the proliferation and
\end{abstract}

Correspondence to: Professor Delian Ren, Department of Immunology, College of Basic Medicine, Southwest Medical University, 25 Taiping Street, Jiangyang, Luzhou, Sichuan 646000, P.R. China

E-mail: rendelian0605@sina.com

Professor Tianyang Dai, Department of Thoracic Surgery, The Affiliated Hospital of Southwest Medical University, 25 Taiping Street, Jiangyang, Luzhou, Sichuan 646000, P.R. China

E-mail: daitianyang0502@sina.com

*Contributed equally

Key words: microRNA-503, proliferation and metastasis, autophagy, protein kinase $\mathrm{A} /$ mammalian target of rapamycin signaling, esophageal squamous cell carcinoma metastasis of ESCC via the activation of autophagy, mediated by the PKA/mTOR signaling pathway.

\section{Introduction}

Esophageal carcinoma (EC) is one of the most common malignancies worldwide, accounting for $>400,000$ cases annualy, with a 5-year survival rate of $20 \%(1,2)$. Unlike in Western countries, esophageal squamous cell carcinoma (ESCC) is the predominant type of cancer in China (3). Epidemiological evidence has indicated that the prognosis of patients with early-stage ESCC is good; however, this sharply declines upon the progression of the disease to an advanced stage. The recurrence and metastasis of ESCC is the pivotal factor leading to the death of patients with advanced disease (4). To date, multiple factors have been reported to be involved in the progression to the advanced stage of $\operatorname{ESCC}(5,6)$; however, the underlying mechanisms remain to be fully elucidated.

Autophagy is a highly conserved catabolic process through which proteins and organelles are recycled to generate intracellular nutrients and energy, to ultimately promote cell survival $(7,8)$. It is well established that autophagy plays dual roles in tumorigenesis, playing either a suppressive or promoting role $(9,10)$. Generally, appropriate autophagy protects tumor cells from hypoxia- or chemotherapy-induced death $(11,12)$. However, excessive activation may trigger autophagic cell death (13). The characteristics of autophagy may be targeted by novel therapeutic strategies aimed at treating tumors. For instance, chloroquine, an inhibitor of autophagy, has been shown to notably enhance the sensitivity of tumor cells to chemotherapeutic drugs $(14,15)$. Moreover, autophagy has also been reported to affect the phenotype of ESCC cells and to be associated with the survival of patients (16).

MicroRNAs (miRNAs or miRs) are a subtype of non-coding RNA molecules with functions in post-transcriptional regulation via binding to the 3'-untranslated regions (UTRs) of targeted mRNAs $(17,18)$. The dysregulation of miRNA expression may lead to the destruction of the equilibrium between 
tumor suppression and promotion (19). To date, multiple miRNA molecules have been confirmed to be implicated in the proliferation, metastasis, apoptosis and chemotherapeutic resistance of ESCC, indicating their crucial role in the development and treatment of ESCC (20-22). hsa-miR-503 belongs to the extended miR-16 family, and is involved in the development of multiple tumors (23). Moreover, increasing evidence has indicated that miR-503 is not only implicated in the regulation of the proliferation and metastasis, but also in the chemosensitivity of tumor cells (24), suggesting that miR-503 may be associated with autophagy. To date, the detailed role of miR-503 in ESCC remains to be defined.

In the current study, we aimed to elucidate the potential mechanisms of action of miR-503, regarding its role in the crosstalk between autophagy and ESCC. Our data indicate that miR-503 inhibits cell proliferation and metastasis by triggering autophagy via the inactivation of PKA signaling in ESCC.

\section{Materials and methods}

Patients and samples. All tissue samples from ESCC patients, who underwent complete surgical resection at the Department of Thoracic Surgery of the Affiliated Hospital of Southwest Medical University (Lu Zhou, China), were collected after obtaining written informed consent. This study was approved by the Ethics Review Board at Southwest Medical University, Luzhou, China. In total, 45 pairs of fresh surgically resected ESCC tissues and matched adjacent non-tumor tissues were collected between 2013 and 2014. Following resection, all the tissues were washed by PBS and frozen by liquid nitrogen immediately, and stored at $-80^{\circ} \mathrm{C}$. In addition, normal esophageal mucosal tissues were obtained from the matched adjacent non-tumor tissues mentioned above, and miR-503 expression in the esophageal mucosa was compared with that in ESCC cell lines. The clinical characteristics of the patients with ESCC are shown in Table I.

Cells and cell culture. The ESCC cell lines, TE-1, Eca109 and Eca9706, were purchased from the Type Culture Collection of the Chinese Academy of Sciences (Shanghai, China) and cultured in RPMI-1640 medium (HyClone, Logan, UT, USA) supplemented with 10\% FBS (BD Biosciences, Franklin Lakes, $\mathrm{NJ}$, USA), at $37^{\circ} \mathrm{C}$ in $5 \% \mathrm{CO}_{2}$.

RNA extraction. TRIzol reagent (Thermo Fisher Scientific, Inc., Waltham, MA, USA) was used to isolate total RNA from the frozen tissues and ESCC cells, according to the manufacturer's instructions. The total RNA was subjected to gel electrophoresis and visualized with an ultraviolet spectrophotometer (Biorad GelDoc XR; Bio-Rad, Hercules, CA, USA) and then stored at $-80^{\circ} \mathrm{C}$.

Reverse transcription-quantitative polymerase chain reaction $(R T-q P C R)$.RT-qPCR assays for protein kinaseCAMP-activated catalytic subunit alpha (PRKACA) and miR-503 expression were performed using a PrimeScript RT reagent kit, SYBR-Green Real-time PCR Master Mix and Permix Ex Taq (both from Takara, Dalian, China), and a TaqMan MicroRNA Reverse Transcription kit (Applied Biosystems, Foster City, CA, USA), respectively, according to the manufacturers' instructions. The parameters for PCR were as follows: Incubation of the templates at $94^{\circ} \mathrm{C}$ for $2 \mathrm{~min}$, followed by 40 cycles of $94^{\circ} \mathrm{C}$ for $20 \mathrm{sec}, 60^{\circ} \mathrm{C}$ for $1 \mathrm{~min}$ and $72^{\circ} \mathrm{C}$ for $20 \mathrm{sec}$, and finally incubation at $72^{\circ} \mathrm{C}$ for $2 \mathrm{~min}$. We used U6 and GAPDH as the housekeeping genes for calculating the relative expression of miR-503 and PRKACA mRNA, respectively. The primers used were as follows: U6 forward, 5'-GCGCGT CGTGAAGCGTTC-3' and reverse, 5'-GTGCAGGGTCCGA GGT-3'; PRKACA forward, 5'-GAGCAGGAGAGCGTGAA AGA-3' and reverse, 5'-AGATCTGGATGGGCTGGTCT-3'; GAPDH forward, 5'-CGGAGTCAACGGATTTGGTCG TAT-3' and reverse, 5'-AGCCTTCTCCATGGTGGTGAA GAC-3'. The $2^{-\Delta \Delta C q}$ method (25) was used to evaluate the relative expression levels of the indicated genes.

Cell proliferation assay. The Eca109 and Eca9706 cells were seeded at a density of $2 \times 10^{3}$ per well in 96 -well plates. The cells were transfected with antagomiR-NC $(800 \mathrm{nM})$, antagomiR-503 $(800 \mathrm{nM})$, agomiR-NC $(600 \mathrm{nM})$, agomiR-503 $(600 \mathrm{nM})$ or negative control (100 ng), or co-transfected with agomiR-503 $(600 \mathrm{nM})$ and PRKACA expression vector $(100 \mathrm{ng})$, respectively. After $48 \mathrm{~h}$, cell proliferation was analyzed using a Cell Counting kit-8 (CCK-8; Beyotime Institute of Biotechnology, Beijing, China) and the Cell-Light ${ }^{\mathrm{TM}}$ EdU Apollo 567 in vitro kit (EdU; Guangzhou RiboBio, Guangzhou, China), according to the instructions provided by the respective manufacturers.

Cell migration and invasion assays. A total of $1 \times 10^{6}$ cells $/ \mathrm{ml}$ of the indicated cells were prepared following transfection with antagomiR-NC (800 nM), antagomiR-503 (800 nM), negative control (100 nM) or siRNA against PRKACA (si-PRKACA; $100 \mathrm{nM}$ ) for $24 \mathrm{~h}$, respectively, and $5 \times 10^{5}$ cells $/ \mathrm{ml}$ of the indicated cells were prepared following transfection with agomiR-NC $(600 \mathrm{nM})$ or agomiR-503 $(600 \mathrm{nM})$, or co-transfection with agomiR-503 $(600 \mathrm{nM})$ and PRKACA vector $(100 \mathrm{ng})$. The migration and invasion of the cells were analyzed using a QCM Laminin Migration assay (ECM220) and a Cell Invasion Assay kit (ECM 550) (both from Merck Millipore, Merck KGaA, Darmstadt, Germany) according to the manufacturer,s instructions. In brief, $1 \times 10^{5}$ indicated cells were suspended in $200 \mu \mathrm{l}$ medium without fetal bovine serum and added into the upper of the chambers. Subsequently, $500 \mu \mathrm{l}$ medium with $10 \%$ fetal bovine serum were added to the lower chambers. The chambers were cultured in the atmosphere with $37^{\circ} \mathrm{C}$ and $5 \% \mathrm{CO}_{2}$ for $24 \mathrm{~h}$ and fixed by $4 \%$ formaldehyde. The chambers were then stained by Giemsa buffer provided with the kit and the cells on the upper part of the membrane were wiped off. We observed the membrane under a light microscope at X100 magnification and 5 random fields were selected for the calculation of the cell numbers.

Cell cycle analysis by flow cytometry. For cell cycle analysis, we treated the cells according to the instructions provided with the BD Cycletest ${ }^{\mathrm{TM}}$ Plus DNA Reagent kit (BD Biosciences). The cell debris and fixation artifacts were gated out, and the cell populations that were at the G0/G1, S and G2/M phases were quantified using Modfit software.

Ad-mRFP-GFP-LC3B transfection. Ad-mRFP (red fluorescent protein)-GFP (green fluorescent protein)-LC3B was 
Table I. The clinical characteristics of the 45 patients with ESCC.

\begin{tabular}{|c|c|}
\hline Index & No. \\
\hline \multicolumn{2}{|l|}{ Sex } \\
\hline Male & 21 \\
\hline Female & 24 \\
\hline \multicolumn{2}{|l|}{ Age (years) } \\
\hline$\leq 60$ & 35 \\
\hline$\geq 60$ & 10 \\
\hline \multicolumn{2}{|l|}{ Tumor size } \\
\hline$\leq 5 \mathrm{~cm}$ & 32 \\
\hline$\geq 5 \mathrm{~cm}$ & 13 \\
\hline \multicolumn{2}{|l|}{ Location } \\
\hline Middle & 27 \\
\hline Lower & 18 \\
\hline \multicolumn{2}{|c|}{ Differentiation } \\
\hline Well & 18 \\
\hline Moderate & 21 \\
\hline Poor & 6 \\
\hline \multicolumn{2}{|c|}{ Invasion depth } \\
\hline Outer layer & 16 \\
\hline Inner layer & 29 \\
\hline \multicolumn{2}{|c|}{ Lympho-node metastasis } \\
\hline Yes & 11 \\
\hline No & 34 \\
\hline \multicolumn{2}{|l|}{ TNM stage } \\
\hline I & 10 \\
\hline II & 15 \\
\hline III & 9 \\
\hline IV & 11 \\
\hline
\end{tabular}

transfected into the indicated cells at a multiplicity of infection (MOI) of 20, and cultured for $72 \mathrm{~h}$ in an environment at $37^{\circ} \mathrm{C}$ and $5 \% \mathrm{CO}_{2}$. DAPI (Beyotime Institute of Biotechnology) was added to the ESCC cells for nuclear visualization before imaging.

In vivo tumorigenesis assay. Female $\mathrm{BALB} / \mathrm{c}-\mathrm{nu}$ mice $(\mathrm{n}=18$, 5-6 weeks of age, weighing 18-20 g) were purchased and kept in barrier facilities on a 12-h light/dark cycle. All experimental procedures were approved by the Institutional Animal Care and Use Committee of Southwest Medical University. The indicated Eca109 cells were injected subcutaneously into the flanks of each mouse $\left[2 \times 10^{6}\right.$ cells suspended in $200 \mu \mathrm{l}$ sterile phosphate-buffered saline (PBS); agomiR-NC left flank and agomiR-503 right flank]. We ensured the survival of all mice on the second day. We cleaned the cages and replaced the water and food every 3 days. Moreover, we observed xenograft tumor growth every 3 days. In the subcutaneous injection groups, 6 weeks later, all mice were anesthetized with $400 \mathrm{mg} / \mathrm{kg}$ chloral hydrate and sacrificed by cervical dislocation, and tumors were dissected and sectioned ( $5 \mathrm{~nm}$ in thickness), followed by
H\&E or immunohistochemistry (IHC; immunofluorescence staining). The volumes of the tumors were calculated using the following formula: Volume $=$ length $\mathrm{x}$ width ${ }^{2} \times 0.5$.

In vivo metastasis assay. For the in vivo pulmonary metastasis assays, $1 \times 10^{6}$ Eca109 cells transfected with agomiR-NC $(600 \mathrm{nM})$ or agomiR-503 $(600 \mathrm{nM})$ were suspended in $200 \mu \mathrm{l}$ of PBS for each mouse. The cells were injected into nude mice (5-6 weeks-old BALB/c-nu; 6 mice were injected with agomiR-NC and 6 mice with agomiR-503) through the lateral tail vein. This experiment was repeated 3 times, with 12 mice used each time. The mice were sacrificed after 6 weeks, and each lung tissue sample was dissected and fixed with $10 \%$ phosphate-buffered neutral formalin overnight prior to paraffin-embedding. The paraffin blocks were then cut into 5 sections and stained with hematoxylin and eosin (H\&E); the sections containing metastatic cells were included to calculate and analyze the number and size of the metastatic nodules.

$H \& E$ staining. The fixed lung tissues from the nude mice, embedded in paraffin, were cut into $4-\mu \mathrm{m}$-thick sections and stained with H\&E (Beyotime Institute of Biotechnology). The slides were photographed under a light microscope (Nikon Corp., Tokyo, Japan).

Immunofluorescence staining. The xenograft tumor tissues were dissected and fixed with $10 \%$ phosphate-buffered neutral formalin overnight prior to paraffin embedding. The paraffin blocks were then cut into sections and incubated with rabbit anti-human cyclin D1 (ab134175), cyclin E1 (ab71535), p-Rb (ab184796) (Abcam, Cambridge, UK), and E-Cadherin (AF0138) and Vimentin (AF0318) antibodies (Beyotime Institute of Biotechnology) (all diluted 1:500).

Confocal and transmission electron imaging. The indicated tumor cells were cultured on glass-bottomed culture dishes. Following transfection with agomiR-503 or agomiR-NC, the indicated cells were washed with PBS and fixed in cold $4 \%$ formaldehyde for $15 \mathrm{~min}$. The cells were then incubated for $10 \mathrm{~min}$ at room temperature in a blocking solution (2\% BSA in PBS) twice. After 3 washes with PBS, the cells were stained with DAPI for $10 \mathrm{~min}$ at room temperature. Images were obtained under a confocal fluorescence microscope (SP8; Leica, Wetzlar, Germany). DAPI was used to visualize the nuclei.

For transmission electron imaging, the indicated tumor cells were collected and washed twice with pre-cooled PBS, and then fixed in $2.5 \%$ glutaraldehyde for $24 \mathrm{~h}$. Subsequently, the samples were sent to FuCheng Biological Technology Co., Ltd. (Shanghai, China) for transmission electron imaging.

Prediction of target gene of miR-503. The databases TargetScan (http://www.targetscan.org/mamm_31/) and miRwalk (http:// zmf.umm.uni-heidelberg.de/apps/zmf/mirwalk/index.html) were used to predict the target of miR-503.

Vector construction. All the plasmids were constructed by Hanbio Biotechnology Co. Ltd. (Shanghai, China). In brief, the DNA oligonucleotides containing the wild-type 3'-UTR of PRKACA or the mutant 3'-UTR of PRKACA were synthesized 
with flanking SpeI and HindIII restriction enzyme digestion sites, respectively. The DNA sequences were connected to pMIR-REPORT vectors to build the luciferase reporter vectors. For the construction of the PRKACA vector, homo sapiens full open reading frame cDNA clone for PRKACA was transcribed, and the product was amplified using primers with flanking SpeI and HindIII restriction enzyme digestion sites. The DNA was subsequently inserted into the pcDNA3.1 vector.

Autophagy inhibition by 3-methyladenine (3-MA). 3-MA was purchased from Selleckchem (Houston, TX, USA; cat. no. S2767). We added $67 \mathrm{ml}$ sterile water to $10 \mathrm{mg}$ 3-MA followed by a $600 \mathrm{C}$ water bath for $10 \mathrm{~min}$, and the final concentration of 3-MA was $1 \mathrm{mM}$. This solution was then added to the medium with $10 \% \mathrm{FBS}$ and cutured in $37^{\circ} \mathrm{C}$ and $5 \% \mathrm{CO}_{2}$ for $12 \mathrm{~h}$. The indicated cells were collected for subsequent analysis.

Oligonucleotide transfection. si-PRKACA or negative control were transfected into the cells using the RiboFECT ${ }^{\mathrm{TM}} \mathrm{CP}$ Transfection kit (Guangzhou RiboBio), according to the manufacturer's instructions. AgomiR-NC, agomiR-503, antagomiR-NC or antagomiR-503 were synthesized by Guangzhou RiboBio and transfected using the RiboFECT ${ }^{\text {TM }}$ CP Transfection kit (Guangzhou RiboBio), according to the manufacturer's instructions. Transfection of the luciferase reporter vectors, empty vectors or PRKACA vectors was performed using Lipofectamine ${ }^{\circledR} 2000$ (Thermo Fisher Scientific, Inc.).

Luciferase assay. The 293T cells (The Cell Bank of Type Culture Collection of Chinese Academy of Sciences, Shanghai, China) were transfected with $0.5 \mu \mathrm{g}$ reporter plasmid harboring wild-type PRKACA-3'-UTR or mutant PRKACA-3'-UTR, or Renilla luciferase control vector (pRL-TK; Promega, Madison, WI, USA) using Lipofectamine ${ }^{\circledR} 2000$ (Thermo Fisher Scientific, Inc.). The indicated cells were transfected with agomiR-NC $(600 \mathrm{nM})$ or agomiR-503 $(600 \mathrm{nM})$, respectively. Following transfection for $24 \mathrm{~h}$, the indicated cells were lysed using a dual luciferase reporter assay system, and fluorescence activity was detected with a GloMax 20/20 Luminometer (Promega). The firefly luciferase activity was normalized to that of Renilla luciferase.

Western blot analysis. Total protein was isolated from the cells using RIPA buffer with 1\% PMSF (both from Beyotime Institute of Biotechnology). Subsequently, $1 \times 10^{6}$ indicated cells were lysed in $1 \mathrm{ml}$ RIPA lysate with $1 \%$ PMSF on ice for $30 \mathrm{~min}$, and centrifuged at $12,000 \mathrm{xg}$ for $10 \mathrm{~min}$ at $4^{\circ} \mathrm{C}$. The supernatant were collected and mixed with $5 \mathrm{X}$ loading buffer, followed by a boiling water bath for $5 \mathrm{~min}$. The quantity of the total protein was determined by BCA assay and $20 \mu \mathrm{g}$ protein were loaded into per lane. Western blot analysis was performed using 10 or $15 \%$ SDS-PAGE gels for different proteins. In total, $5 \%$ non-fat dry milk was used for blocking the PVDF membranes. The membranes were incubated with the following primary antibodies: Rabbit anti-human PRKACA monoclonal antibody (dilution, 1:1,000; ab76238), rabbit anti-human cyclin D1 polyclonal antibody (dilution, 1:1,000; ab134175), rabbit anti-human cyclin E1 polyclonal antibody (dilution,1:1,000; ab71535), rabbit anti-human $\mathrm{pRb}$ monoclonal antibody (dilution, 1:1,000; ab184796) (all from Abcam), rabbit anti-human LC3B polyclonal antibody (dilution, 1:1,000, AL221; Beyotime Institute of Biotechnology), rabbit anti-human p62 monoclonal antibody (dilution, 1:1,000; 23214S), rabbit anti-human mTOR polyclonal antibody (dilution, 1:1,000; \#2972), rabbit anti-human phospho-mTOR polyclonal antibody (dilution, 1:1,000; \#2971) (all from Cell Signaling Technology, Danvers, MA, USA), and rabbit anti human E-Cadherin (AF0138) and Vimentin (AF0318) polyclonal antibodies (dilution, 1:1,000; Beyotime Institute of Biotechnology). GAPDH protein served as an internal reference, using mouse anti human GAPDH monoclonal antibody (dilution, 1:5,000; AF0006; Beyotime Institute of Biotechnology). The primary incubation was followed by incubation with goat anti rabbit or mouse HRP-conjugated secondary antibodies (dilution, 1:4,000; A0208 and A0216; Beyotime Institute of Biotechnology) for $1 \mathrm{~h}$ at room temperature. Finally, the results were analyzed by enhanced chemiluminescence. Quantity One software (4.6.2) was used to calculate the relative expression of related proteins.

Statistical analysis. The two-tailed Student's t-test was used to determine the differences between 2 groups. Multi-group measurement data were analyzed by one-way ANOVA followed by a Dunnett's post hoc test. Pearson's correlation analysis was used to analyze the correlation between the expression of miR-503 and PRKACA mRNA. A P-value of $<0.05$ was considered to indicate a statistically significant difference. All statistical analyses were performed using SPSS 17.0 software and graphs were generated using GraphPad Prism 6.0 (Graphpad Software Inc., San Diego, CA, USA).

\section{Results}

miR-503 is highly expressed in ESCC tissues and cell lines. To investigate the clinical relevance of miR-503 expression in the initiation and progression of ESCC, we analyzed 45 freshly-frozen tissue samples of ESCC and 45 matched adjacent non-tumor tissues by RT-qPCR. The results revealed that miR-503 expression in the ESCC tumor tissues was markedly upregulated compared with the adjacent non-tumor tissues (NC) $(\mathrm{P}<0.05$; Fig. 1A). It should be noted that although the expression of miR-503 was markedly upregulated in the ESCC tissues, this was just several folds in comparison with that in the adjacent non-tumor tissues, and not all the patients exhibited a higher miR-503 expression in their tumor tissues. This suggests that if the number of patients with ESCC was expanded, then perhaps a greater number of patients would be found to have a lower expression of miR-503 in their tumor tissue. Further investigations are warranted on this matter.

However, a higher expression of miR-503 was observed in the ESCC tissues without lymph node metastasis compared with those with lymph node metastasis $(\mathrm{P}<0.05$; Fig. 1B). Further statistical analysis revealed that miR-503 expression was inversely associated with the clinical stage (Fig. 1C) and differentiation in patients with ESCC (Fig. 1D). To determine the level of miR-503 expression in ESCC cell lines, we then compared miR-503 expression in the normal esophageal epithelium mucosal tissues from the adjacent non-tumor 
A

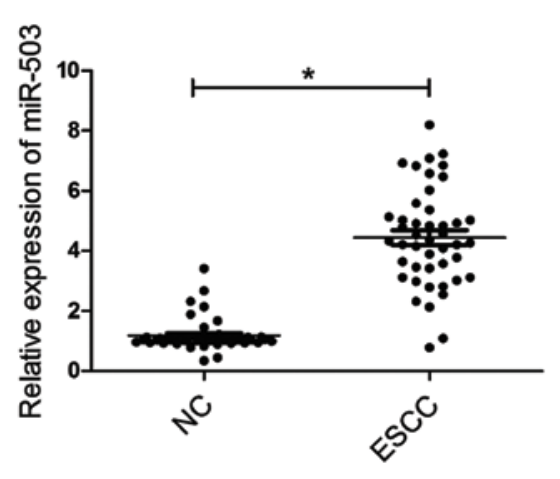

C

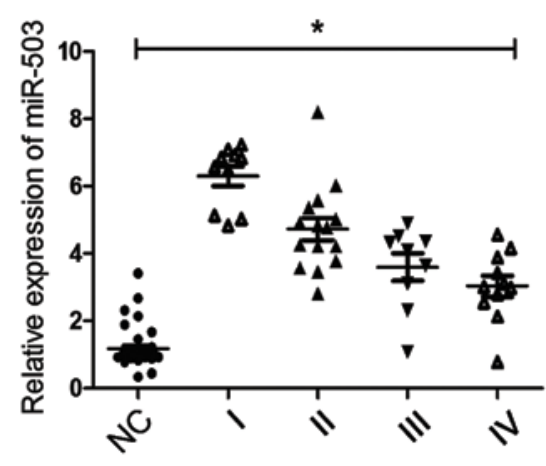

E

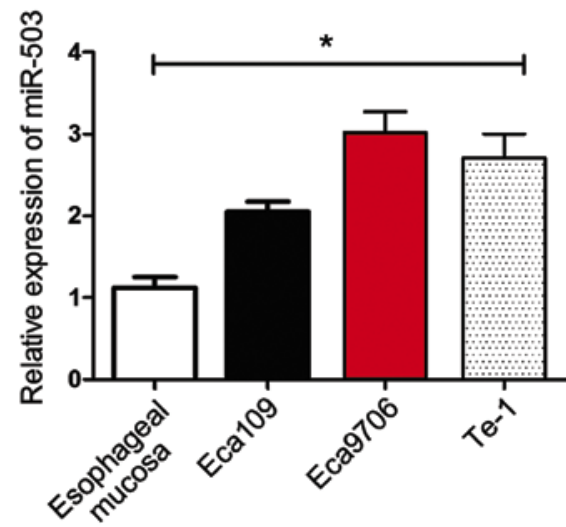

B

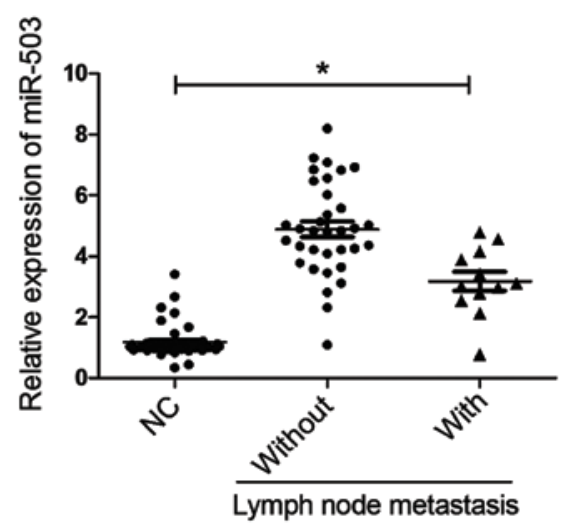

D

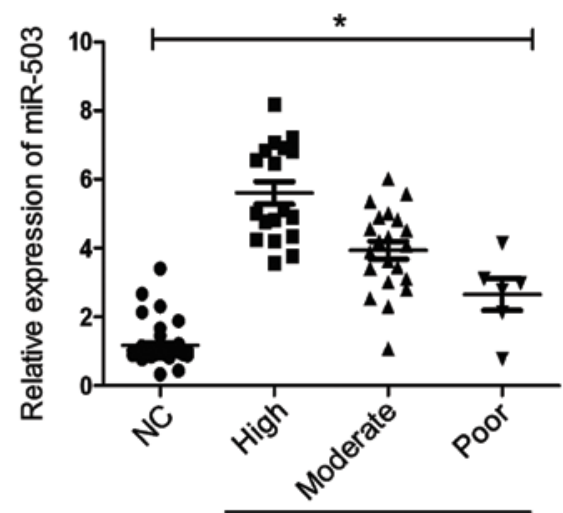

Differentiation

Figure 1. Expression of miR-503 in esophageal squamous cell carcinoma (ESCC) tissues and cell lines and its correlation with clinical characteristics. (A) miR-503 expression was upregulated in ESCC tissues compared to the matched adjacent non-tumor tissues; (B) miR-503 expression was decreased in ESCC tissues with lymph node metastasis compared with tissues without lymph node metastasis. (C) miR-503 expression negatively correlated with (C) the TNM stage and (D) tumor differentiation. (E) miR-503 expression was upregulated in ESCC cell lines. * $\mathrm{P}<0.05$.

tissues of the 45 ESCC samples with that in the ESCC cell lines (Eca109, Eca9706 and TE-1) by RT-qPCR. As expected, the expression of miR-503 was also upregulated in the ESCC cell lines (Fig. 1E). Specifically, Eca9706 and TE-1 exhibited a $>2$-fold increase in miR-503 expression. Taken together, we thus inferred that miR-503 may play a suppressive role in ESCC. As marked changes in miR-503 expression may display its biological function, we therefore further increased the miR-503 expression in the ESCC cells.

miR-503 influences the proliferation and metastasis of ESCC cells in vitro. To determine the biological function of miR-503 in ESCC, we chemically synthesized the sequences of agomiR-503,
antagomiR-503 and a corresponding negative control (NC) in vitro, and then transfected these into the Eca109 and Eca9706 cells. The transfection efficiency was determined by RT-qPCR and the results revealed that the miR-503 expression was markedly decreased or increased (data not shown). In the current study, multiple biological functional analyses of miR-503 were performed, and the results revealed that the ectopic expression of miR-503 in the ESCC cell lines attenuated cell proliferation, as determined by CCK-8 assay (Fig. 2A) and EdU assays (Fig. 2B). Moreover, a cell cycle array performed by flow cytometry revealed that the overexpression of miR-503 markedly increased the percentages of cells in the G1 phase (by 19.05 and $12.20 \%$ in the Eca109 and Eca9706 cells, respectively) and decreased 

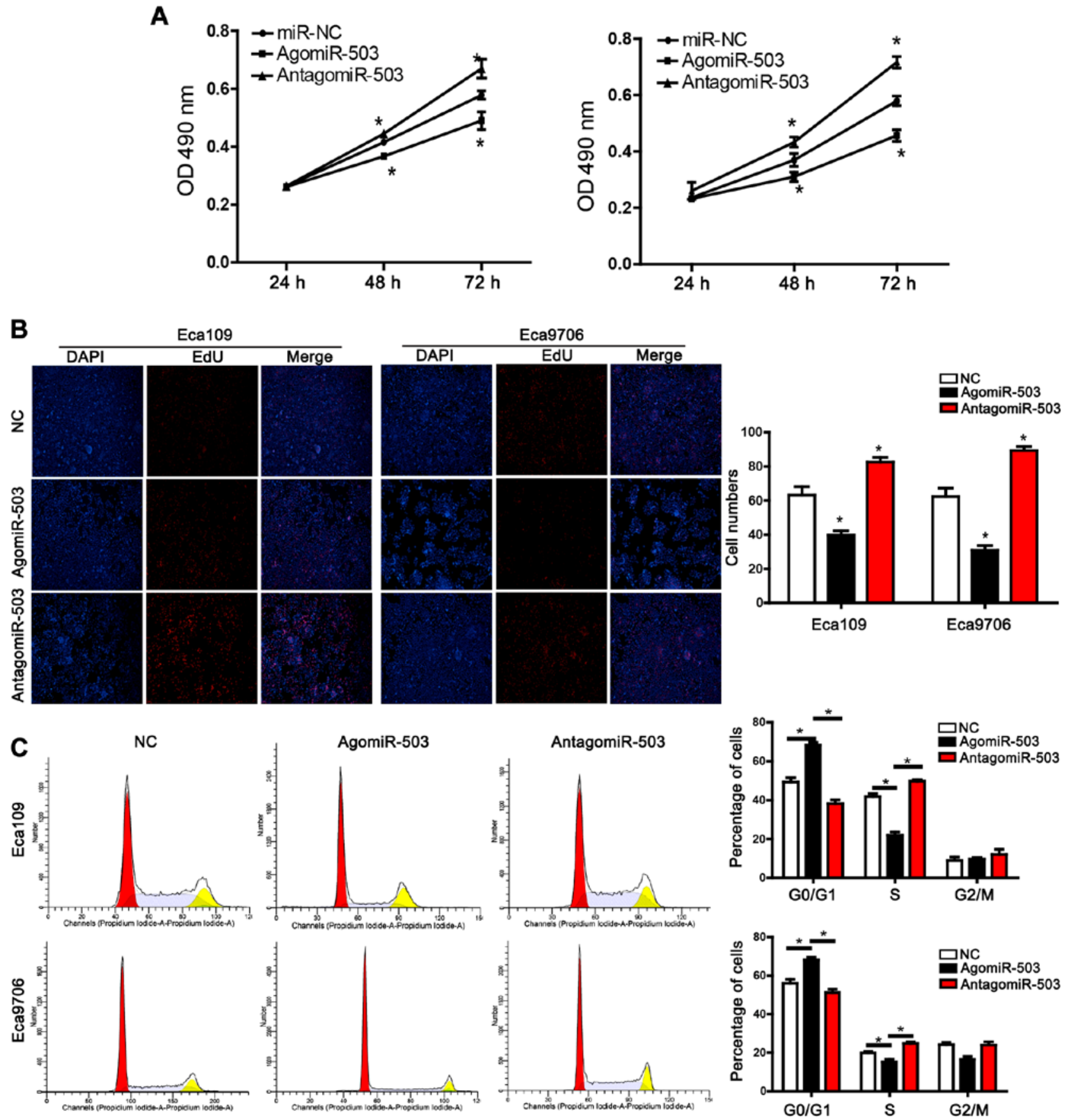

Figure 2. miR-503 inhibits the proliferation of esophageal squamous cell carcinoma (ESCC) cells. (A) A CCK-8 array revealed that the ESCC cells transfected with agomiR-503 or antagomiR-503 exhibited a decreased or increased proliferative capacity, respectively. (B) An EdU assay showed that miR-503 decreased the ESCC cell number in the proliferative phase. (C) Flow cytometry revealed that miR-503 promoted cell cycle arrest at the G1/S phase in ESCC cells. ${ }^{*} \mathrm{P}<0.05$; all experiments were repeated 3 times.

the proportions cells in the S phase (by 19.9 and $4.59 \%$ in the Eca109 and Eca9706, respectively) (Fig. 2C). Moreover, we evaluated whether miR-503 was biologically involved in the migration and invasion of ESCC cells through Transwell assays with or without Matrigel. As expected, transfection with agomiR-503 effectively attenuated the propensity of Eca109 and Eca9706 cells to invade the Matrigel. Similar results were also obtained in the Transwell assay without Matrigel. However, the tumor cells transfected with antagomiR-503 exhibited increased proliferative and metastatic capabilities compared with the cells in the NC group (Fig. 3A and B). These data thus suggest a suppressive role of miR-503 in ESCC.
In addition, we detected a number of biomarkers known to be associated with proliferation and metastasis by western blot analysis. Compared with the negative controls, the cells with a high miR-503 expression (agomiR-503 group) exhibited a decrease in the protein expression of phosphorylated $\mathrm{Rb}$ protein, as well as in the expression of cyclin D1 and cyclin E1. Moreover, decreased Vimentin and increased E-Cadherin expression levels were observed in the ESCC cells transfected with agomiR-503, whereas opposite results were obtained in the group transfected with antagomiR-503 (Fig. 3C). These data thus indicate that miR-503 may play a tumor suppressive role in ESCC by regulating cell proliferation and metastasis. 
A
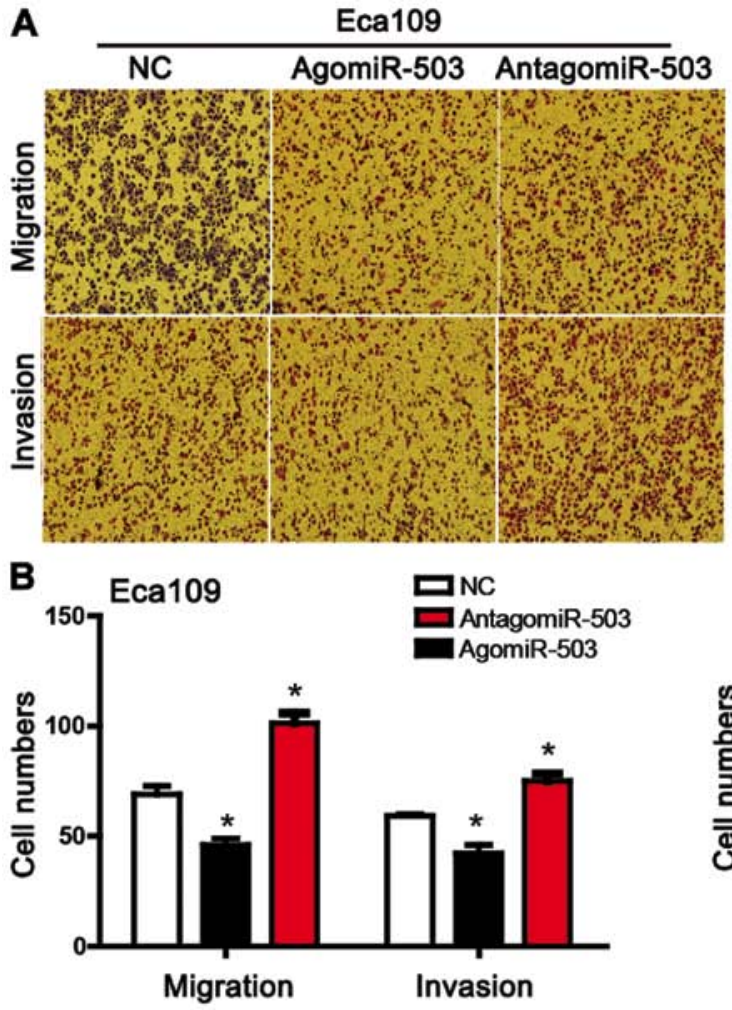

C

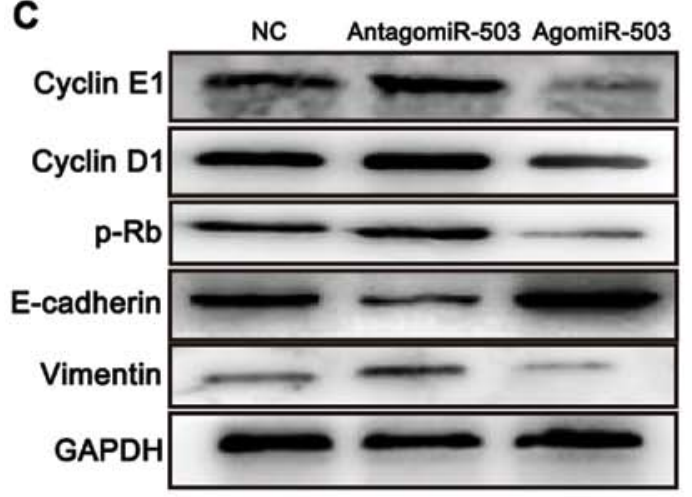

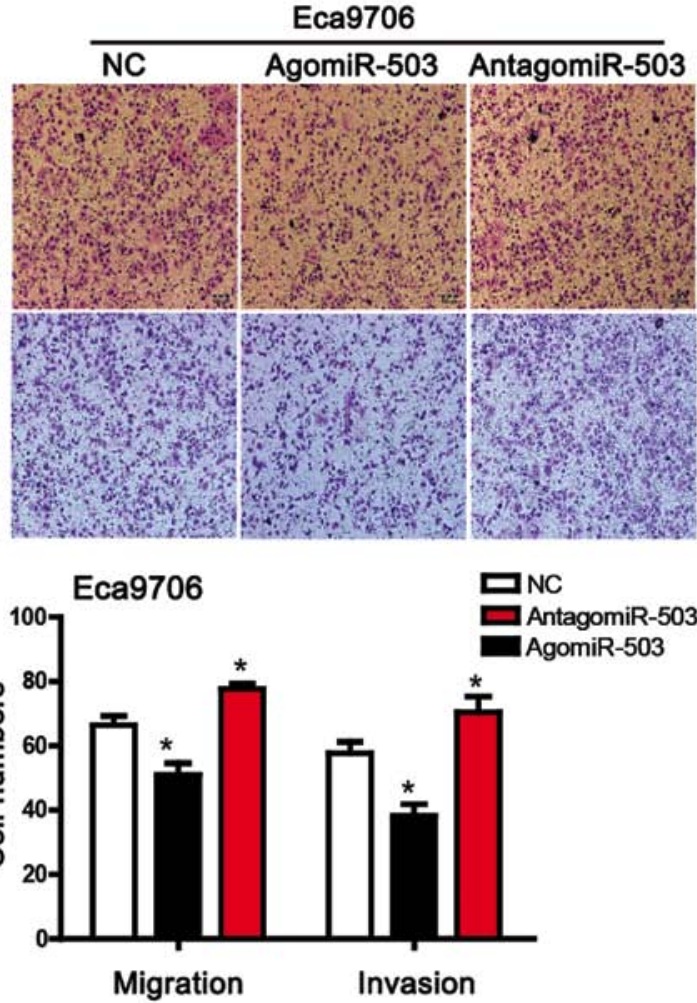

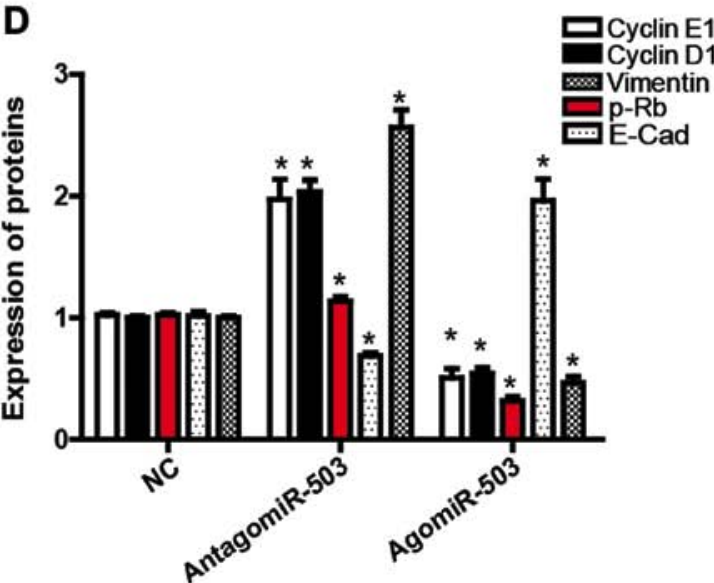

Figure 3. miR-503 suppresses the metastasis of esophageal squamous cell carcinoma (ESCC) cells. (A) Representative images of Transwell assays and (B) the histogram of the statistical results indicated that miR-503 inhibited the migration and invasion of ESCC cells. (C) Western blot analysis was used to detect the protein expression of cyclin D1, cyclin E1, p-Rb, E-Cadherin and Vimentin. * $\mathrm{P}<0.05$; all experiments were repeated 3 times.

miR-503 inhibits ESCC proliferation and metastasis in vivo. To further examine the effects of miR-503 on ESCC growth and invasiveness in vivo, we subcutaneously injected the Eca109 cells transfected with NC or agomiR-503 into the left and right flanks of nude mice. As expected, the injected ESCC cells transfected with miR-503 grew into smaller tumors compared with those in the NC group. The maximum diameter of a single tumor was $1.6 \mathrm{~cm}$, while the minimum was $0.4 \mathrm{~cm}$. The volumes of the tumors formed by ESCC cells transfected with agomiR-503 were significantly smaller than those in the NC group (Fig. 4A). Moreover, the significant downregulation of Vimentin, p-Rb, cyclin D1 and cyclin E1 protein levels, and the upregulation of E-cadherin protein levels, were observed in the agomiR-503 group by IHC (Fig. 4B). For pulmonary metastasis assays, the ESCC cells transfected with agomiR-503 or the negative control cells were injected into nude mice through the lateral tail vein. All mice were sacrificed after 6 weeks, and the lungs were removed and subjected to histological and pathological examination. Accordingly, the agomiR-503 group exhibited a marked decrease in metastatic burden in the lungs of nude mice compared with the negative controls (Fig. 4C). Taken together, these results indicated that miR-503 exerted an inhibitory effect on ESCC development.

miR-503 triggers autophagy in ESCC cells. Studies have demonstrated that miR-503 can regulate the chemosensitivity of multiple types of cancer, indicating that miR-503 may be involved in intracellular autophagy $(24,26)$. Therefore, we examined the level of autophagy in the Eca109 and Eca9706 cells in the agomiR-503 and negative control groups. We 

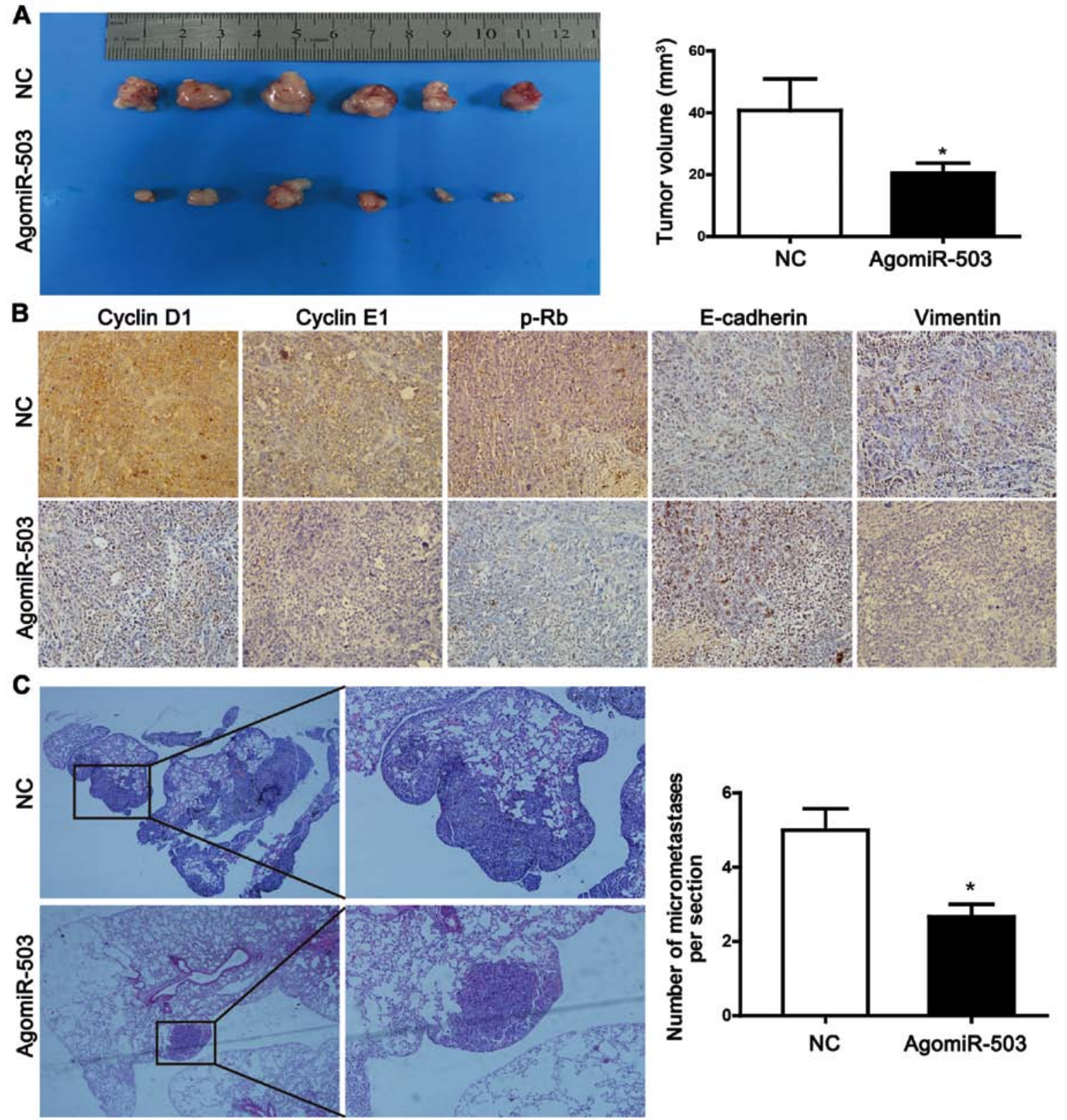

Figure 4. miR-503 inhibits the proliferation and metastasis of esophageal squamous cell carcinoma (ESCC) in vivo. (A) miR-503 inhibited the growth of ESCC cells in vivo. (B) Immunohistochemistry was used to detect the expression of cyclin D1, cyclin E1, p-Rb, E-Cadherin and Vimentin in xenograft tumor tissues. (C) miR-503 inhibited the metastasis of ESCC cells in vivo. ${ }^{*} \mathrm{P}<0.05$

first infected the indicated cells with Ad-mRFP-GFP-LC3B (MOI of 20) to monitor the formation of autophagosomes. The results revealed that more autophagosomes were formed in the agomiR-503 group, as determined by laser scanning confocal microscopy (Fig. 5A and C). We also confirmed autophagosome formation under a transmission electron microscope (Fig. 5B). Moreover, we found that the protein level of LC3-II, a marker of autophagy, was markedly increased, whereas that of p62, a marker of autophagic flux, was decreased following transfection with agomiR-503 (Fig. 5D-F). These data thus indicate that miR-503 triggers autophagy in ESCC.

miR-503-mediated autophagy affects the proliferation and metastasis of ESCC cells. Increasing evidence has indicated that autophagy plays a dual role in the development of tumors, being promotional or inhibitory $(27,28)$; however, the function of miR-503-induced autophagy in ESCC remains to be determined. Therefore, in this study, we used 3-MA (50 $\mathrm{nM})$ to inhibit miR-503-mediated autophagy, and detected the biological function of the indicated ESCC cells. The results of CCK-8 and Transwell assays revealed that 3-MA partially 'rescued' the proliferation and metastasis of ESCC cells mediated by miR-503 (Fig. 6), indicating that miR-503-triggered autophagy is implicated in the proliferation and metastasis of ESCC cells.

PRKACA is a novel target of miR-503. It is well established that the function of miRNAs in tumor development is dependent on their target genes, and that individual miRNAs can repress the translation of multiple mRNAs. Therefore, it was considered of crucial importance to identify the key target genes of miR-503. We thus analyzed the potential target 
A
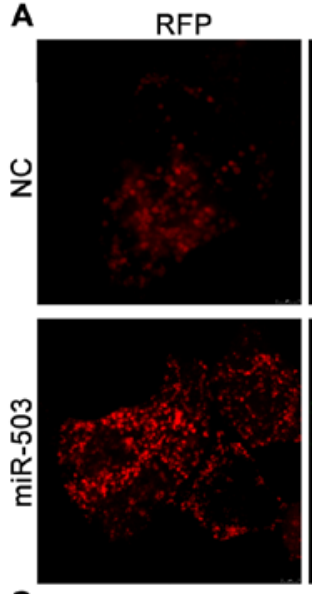

C

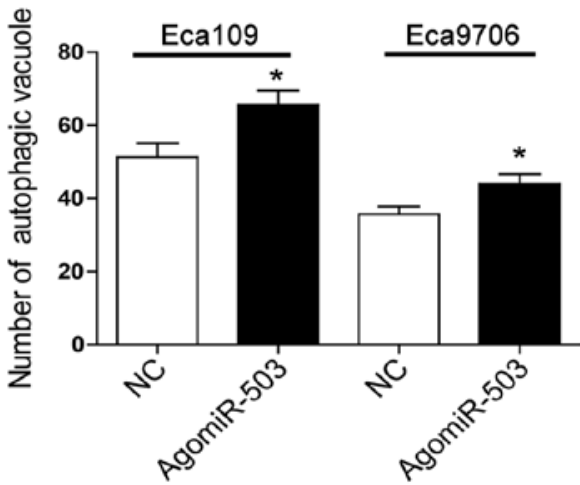

E

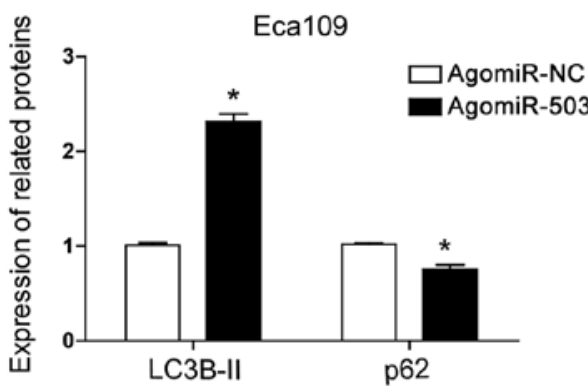

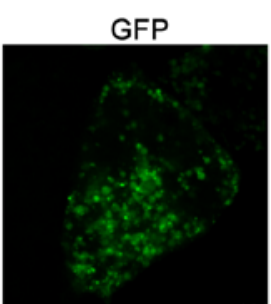

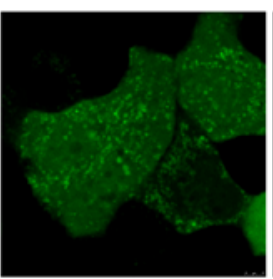

Eca9706

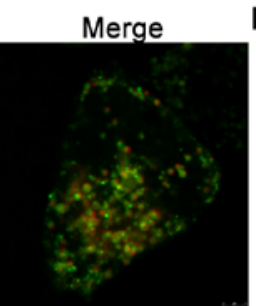

B Original

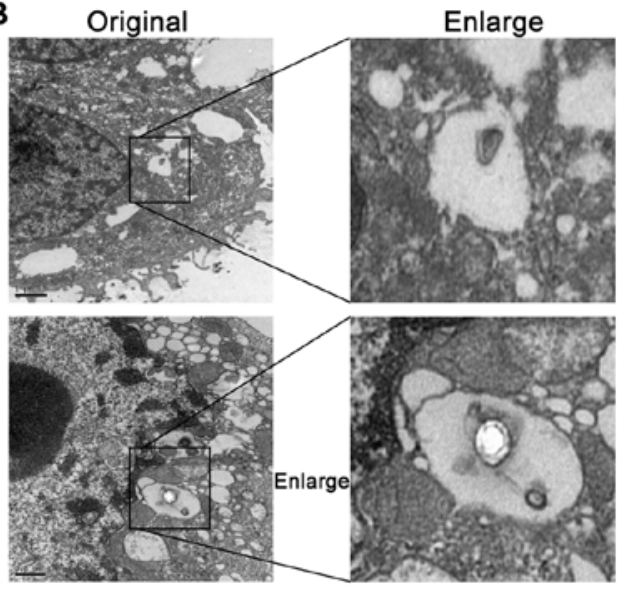

D
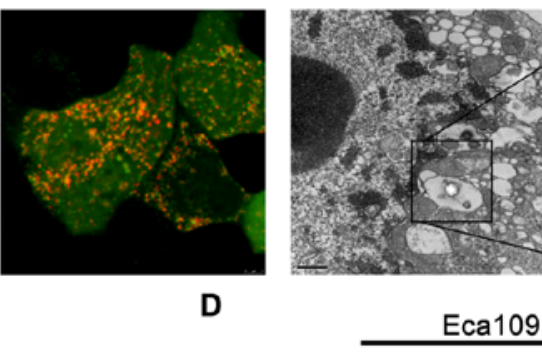

Eca9706

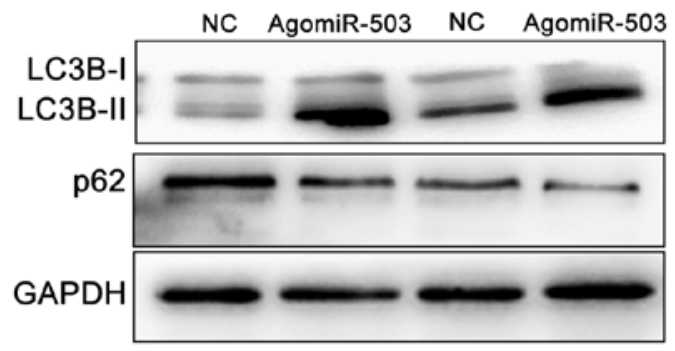

F

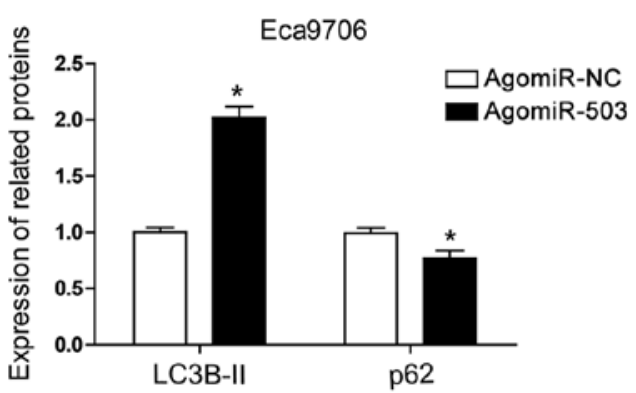

Figure 5. miR-503 activates autophagy in esophageal squamous cell carcinoma (ESCC) cells. (A) Confocal laser scanning revealed that autophagosome formation in ESCC cells transfected with agomiR-503 was enhanced. (B) Electron microscopy confirmed this autophagosome formation in ESCC cells. (C) The histogram indicates the numbers of autophagosomes in the indicated cells. (D) Western blot analysis was used to detect autophagy-related protein expression following agomiR-503 transfection. Histograms (E and F) were used to show the statistical results of western blot analysis of the indicated ESCC cells. ${ }^{*}<0.05$; all experiments were repeated 3 times.

genes using the online bioinformatics tools, TargetScan and miRwalk. We found that RKACA, closely associated with tumor proliferation and metastasis, harbored the binding site of miR-503 (Fig. 7A). We then examined whether PRKACA was downstream of miR-503, and found that PRKACA mRNA and protein expression levels both decreased in the presence of ectopic miR-503 expression (agomiR-503); this effect was reversed by transfection with antagomiR-503 in vitro (Fig. $7 \mathrm{~B}$ and $\mathrm{C}$ ). To further confirm that $\mathrm{PRKACA}$ is a target gene of miR-503, we constructed vectors harboring the wild-type or mutant 3'-UTR of PRKACA mRNA, individually fused directly downstream of the firefly luciferase gene for luciferase assays. We co-transfected the plasmid harboring wild-type or mutant PRKACA with agomiR-NC and agomiR-503, respectively, into 293T cells, and found that the ectopic miR-503 expression significantly decreased the relative luciferase activity of the wild-type 3'-UTR of PRKACA
$(\mathrm{P}<0.01)$, whereas the luciferase activity of the mutant 3'-UTR was not significantly altered (Fig. 7D). Moreover, we found a negative correlation between PRKACA mRNA and miR-503 expression by RT-qPCR in 15 ESCC tissues (Fig. 7E). Taken together, miR-503 was found to be able to directly regulate PRKACA expression in ESCC.

PRKACA restores the malignant phenotype of ESCC cells suppressed by miR-503. PRKACA, a critical regulator of the PKA signaling pathway, has been reported to be implicated in the development of multiple tumors $(29,30)$; however, its function in ESCC remains unclear. First, we evaluated whether the silencing or overexpression of PRKACA could mimic the biological effects of miR-503 on the growth and metastasis of ESCC cells. Western blot analysis revealed that PRKACA expression was markedly down- or upregulated in the indicated groups (Fig. 7F). Moreover, as was expected, the proliferative 
A

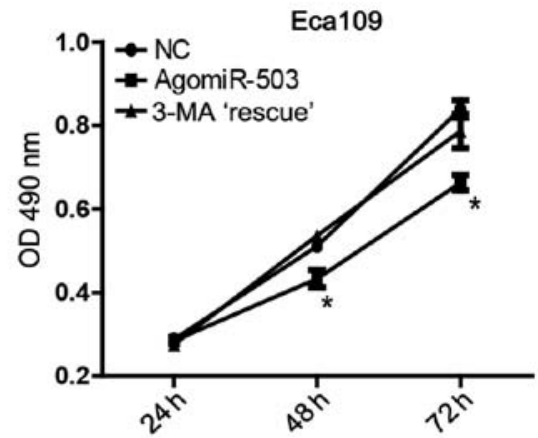

B
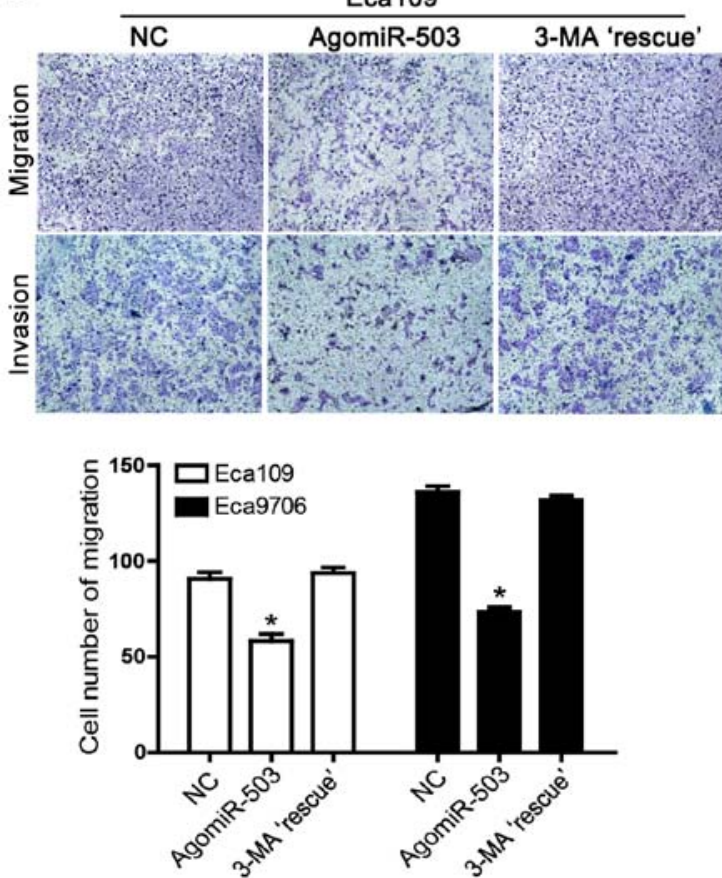

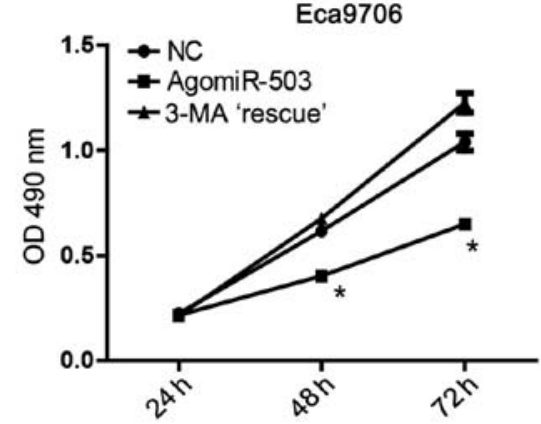

Eca9706
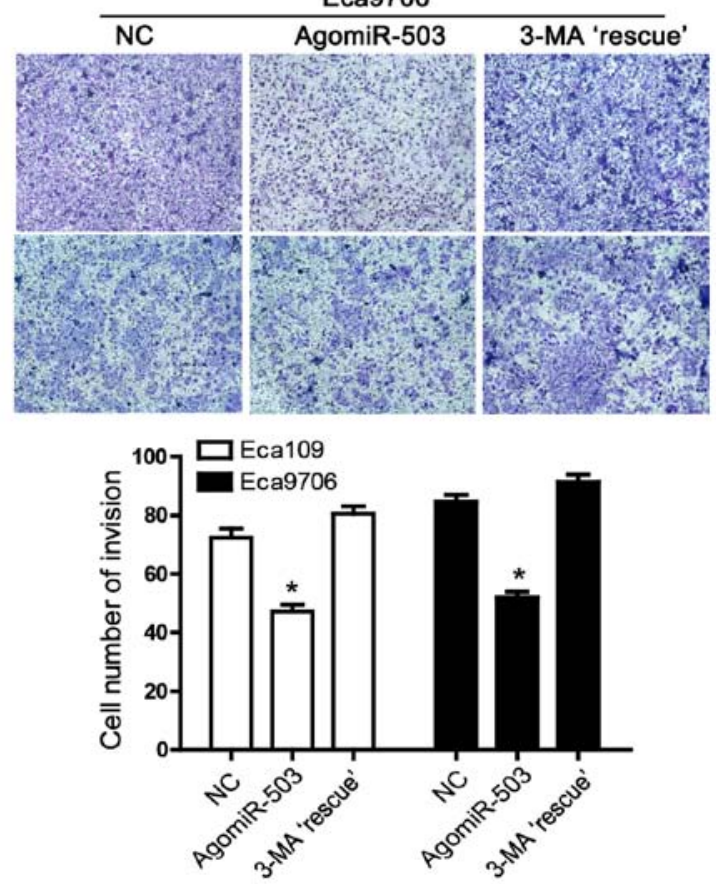

Figure 6. Autophagy inhibition 'rescues'/restores the aggressive phenotype of esophageal squamous cell carcinoma (ESCC) cells. (A) A CCK-8 assay revealed that an autophagy inhibitor (3-MA) restored the proliferative capacity of the cells which was inhibited by miR-503. (B) A Transwell assay revealed that the metastatic ability which was suppressed by miR-503 was restored by 3-MA. ${ }^{*} \mathrm{P}<0.05$; all experiments were repeated 3 times.

and metastatic capabilities of the ESCC cells were markedly impaired with PRKACA silencing, or enhanced with PRKACA upregulation (Fig. 7G and $\mathrm{H}$ ), indicating that PRKACA promoted the proliferation and metastasis of ESCC. Taken together, our data indicate that miR-503 suppresses the proliferative and metastatic potential of ESCC cells by targeting PRKACA.

miR-503 influences cellular autophagy by targeting PRKACA. In this study, we revealed that miR-503 regulated the autophagy of ESCC; however, whether PRKACA was involved in this process remained unclear. Thus, we first investigated whether PRKACA was involved in autophagy in ESCC cells, and found that PRKACA inhibited the autophagy of ESCC cells, as shown by confocal laser scanning microscopy and western blot analysis (Fig. 8A and B). We further upregulated PRKACA in the ESCC cells transfected with agomiR-503 as the 'rescue' group, in order to evaluate its effect on autophagy mediated by miR-503. Western blot analysis revealed that the expression of LC3BII and p62 was restored (to normal levels) in the agomiR-503 + PRKACA vector group ('rescue' group) (Fig. 8C and D). These data suggest that PRKACA is involved in the autophagy induced by miR-503.
Recently, the mammalian target of rapamycin (mTOR) complex, a well-known regulator of autophagy, was reported to be closely associated with PKA. Therefore, we examined the association between PRKACA and mTOR in order to clarify the underlying mechanisms. As expected, the down- or upregulation of PRKACA attenuated or enhanced the phosphorylation of mTOR, respectively; moreover, PRKACA restored the phosphorylation of mTOR in the agomiR-503 + PRKACA vector group ('rescue' group), which was suppressed by miR-503 (Fig. 8B-D). Taken together, we concluded that miR-503 promoted autophagy by inhibiting the PKA-mTOR pathway.

PRKACA 'rescues' the proliferation and metastasis of $E S C C$. To further explore whether the biological function of miR-503 could be restored by PRKACA in ESCC, we co-transfected the PRKACA vector with agomiR-NC or agomiR-503, respectively, for the 'rescue' assay. The results revealed that the proliferation and metastasis of the ESCC cells transfected with agomiR-503 was restored by PRKACA [agomiR-503 + PRKACA vector group ('rescue' group)] (Fig. 9). Taken together, PRKACA restored the 
A

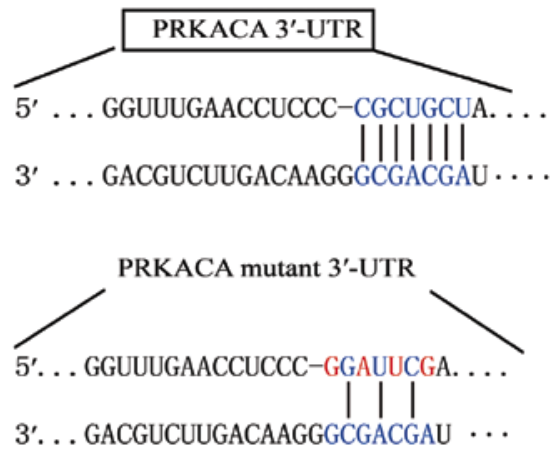

C

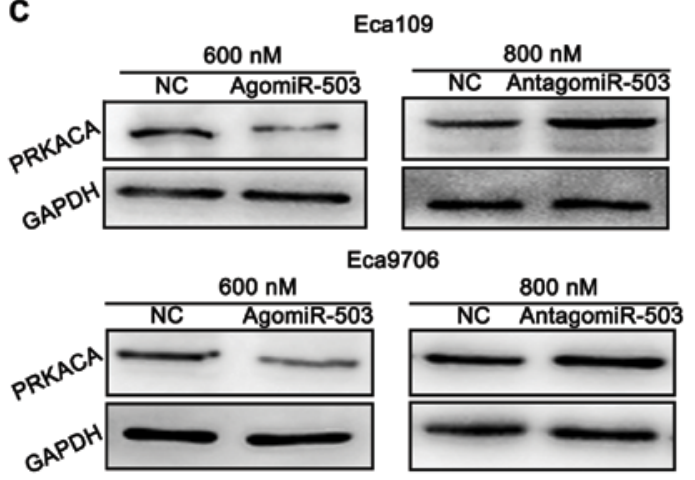

E

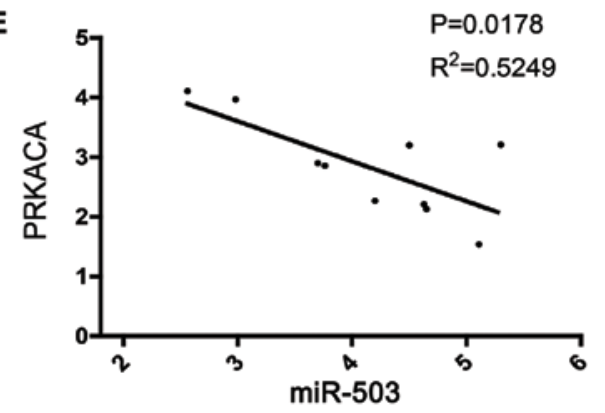

G

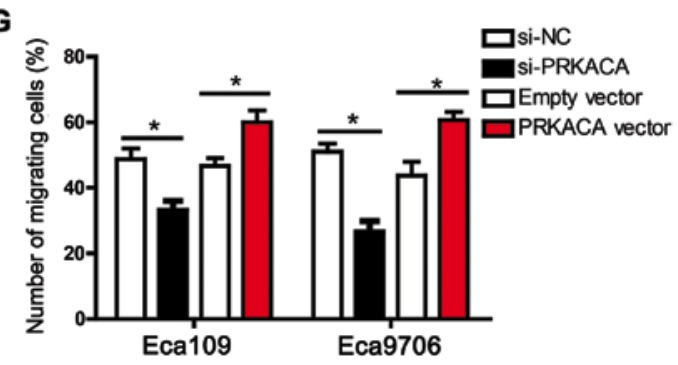

B

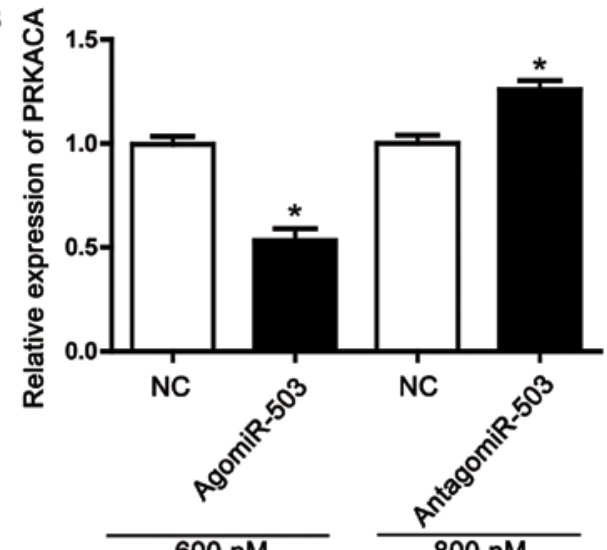

D

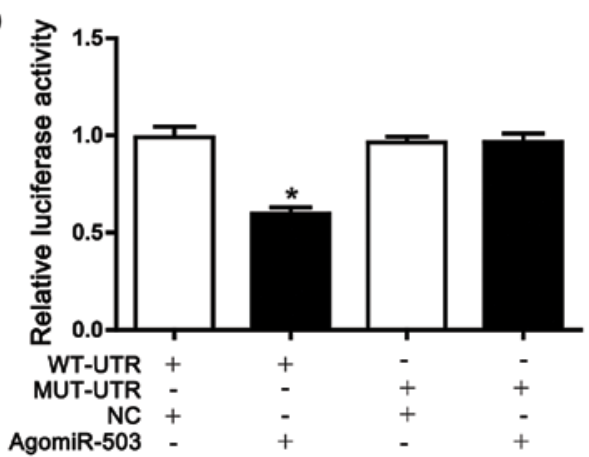

F

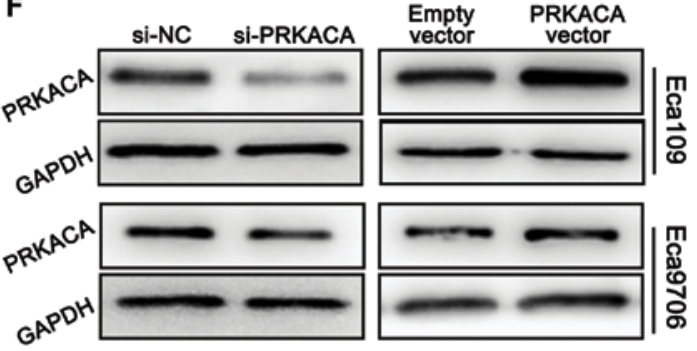

H

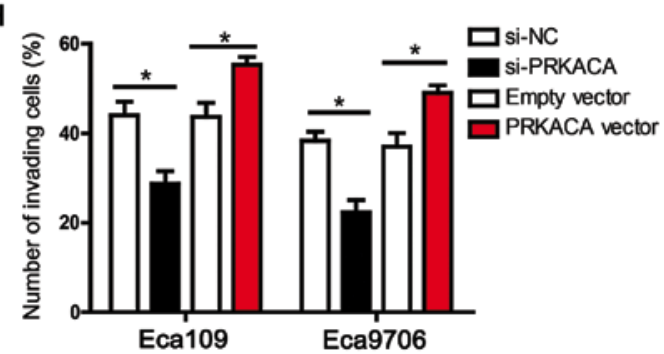

Figure 7. PRKACA is a target gene of miR-503 in esophageal squamous cell carcinoma (ESCC). (A) Graph indicating the wild-type and mutant binding site of miR-503 to PRKACA, predicted by bioinformatics. (B) RT-qPCR and (C) western blot analysis were used to detect the relative expression of PRKACA following agomiR-503 or antagomiR-503 transfection. (D) A histogram was used to show the results of the dual luciferase reporter assay. (E) A histogram was used to show the correlation between miR-503 and PRKACA expression. (F) Western blot analysis was used to investigate the efficiency of PRKACA silencing or overexpression. A histogram was used to indicated the influence of PRKACA on $(\mathrm{G})$ the migration and $(\mathrm{H})$ invasion of ESCC cells. "P<0.05; all experiments were repeated 3 times.

proliferation and metastasis of ESCC which had been suppressed by miR-503.

Illustrating the mechanisms of action of miR-503 in ESCC. In order to better elucidate the underlying mechanisms through which miR-503 regulates the malignant phenotype in ESCC, we created a diagram with which to describe the mechanisms through which miR-503 inhibits the proliferation and metastasis of ESCC by triggering autophagy via PKA/mTOR signaling (Fig. 10).

\section{Discussion}

In the current study, we identified the suppressive role of miR-503 in the regulation of the ESCC malignant phenotype through its targeting of PRKACA, and revealed that the 
A
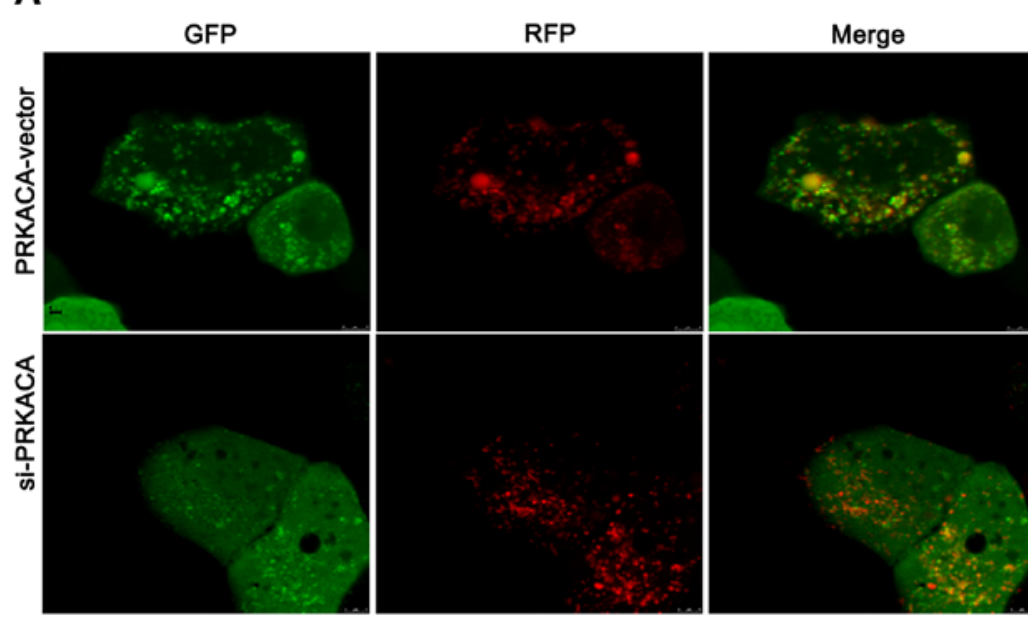

B
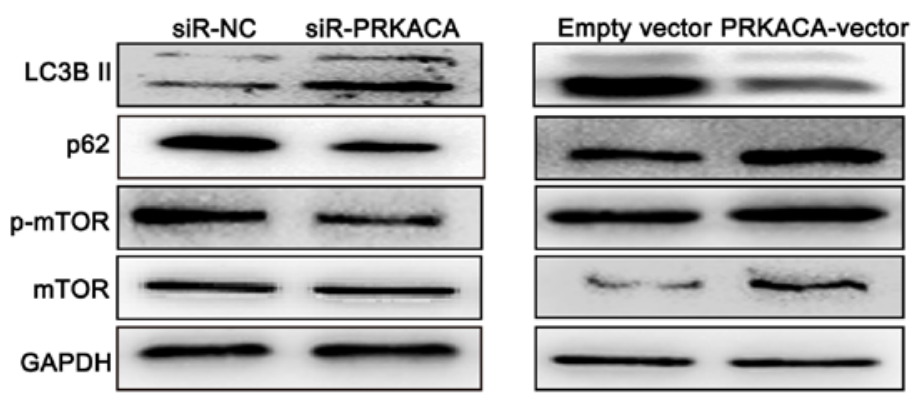

D
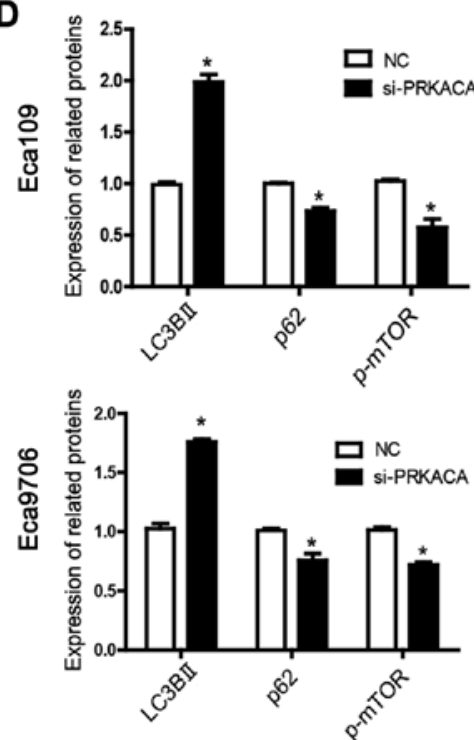
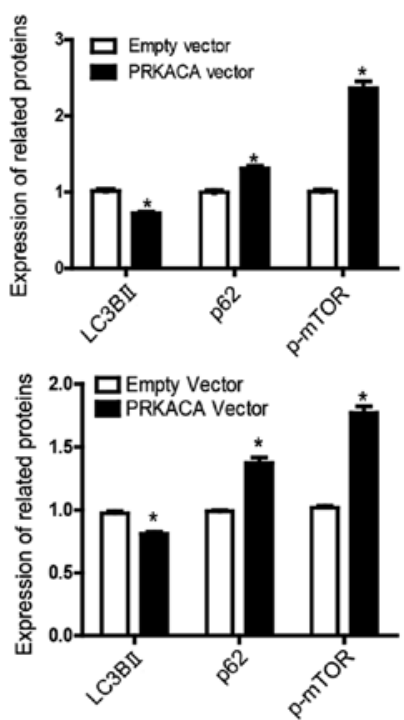

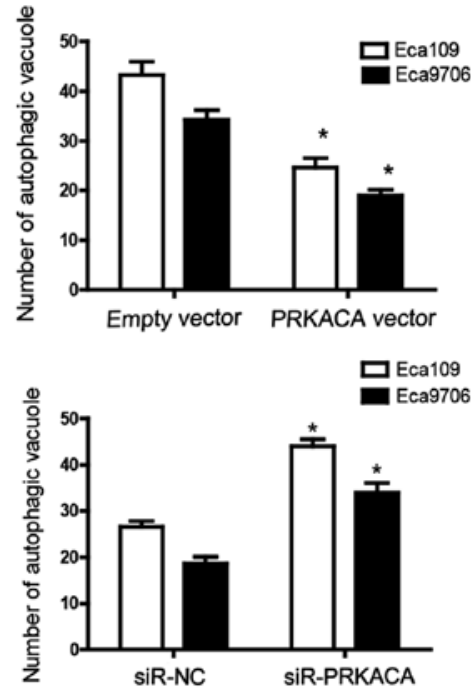

C
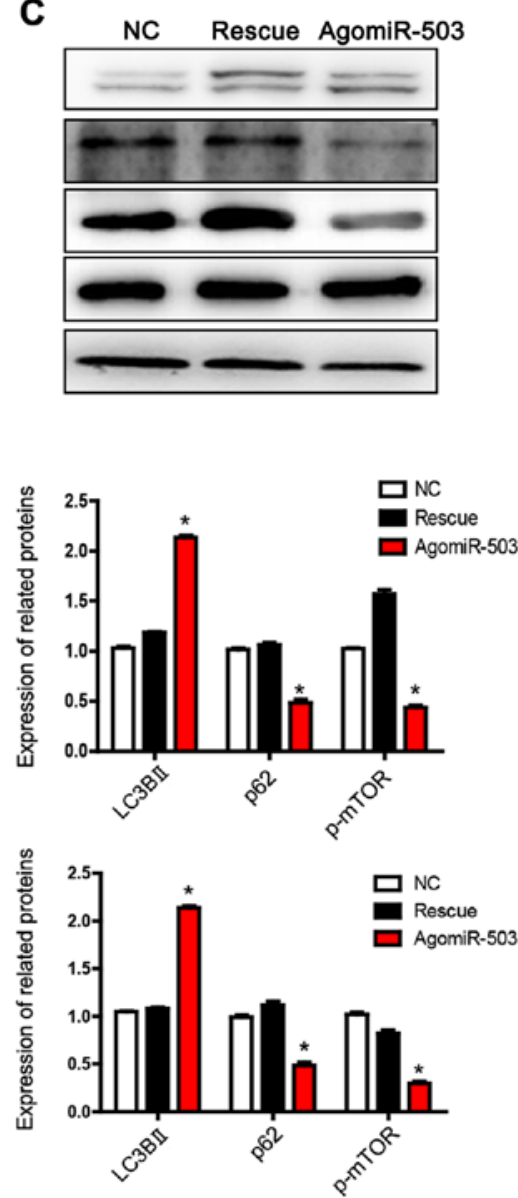

Figure 8. PRKACA inhibits the autophagy of esophageal squamous cell carcinoma (ESCC) cells. (A) Confocal laser scanning microscopy was used to examine the effects of PRKACA on autophagosome formation in ESCC cells. (B) Representative western blots of the expression levels of LC3B-II, p62 and phosphorylated mTOR in the indicated ESCC cells and (C) the restorative effect of PRKACA on the protein expression levels, following miR-503-associated upregulation. (D) Histograms were used to show the statistical results of western blot analysis. The 'rescue' group represents the ESCC cells that were cotransfected with agomiR-503 and PRKACA vector. ${ }^{*} \mathrm{P}<0.05$; all experiments were repeated 3 times.

underlying mechanisms through which miR-503 induced autophagy to inhibit the proliferation and metastasis of ESCC involved the inactivation of PKA/mTOR signaling.

mir-503 is a member of the miR-16 family and has been reported to be associated with carcinogenesis; its aberrant expression has been observed in multiple tumors $(31,32)$. While certain studies have observed that miR-503 is downregulated in several types of cancer, such as oral cancer, hepatocellular carcinoma, non-small cell lung cancer and endometrioid endometrial cancer (32-35), it has been observed that miR-503 expression is upregulated in adrenocortical carcinoma, parathyroid carcinoma and retinoblastoma (36-38). In this study, 
A

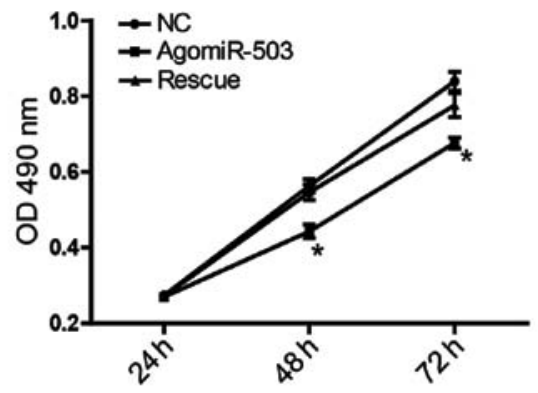

B
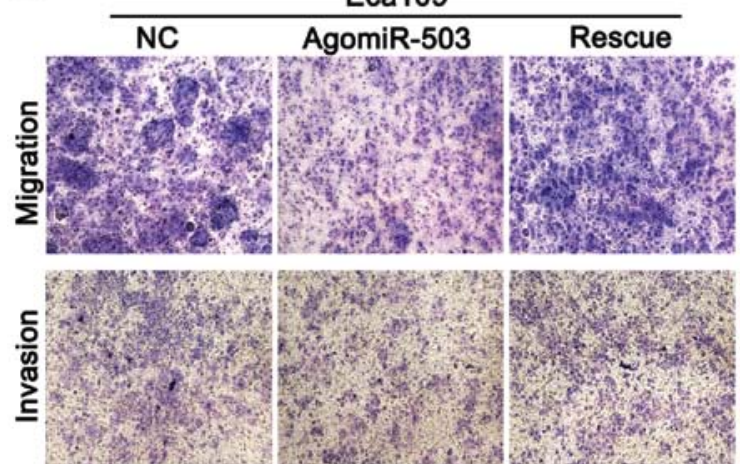

C

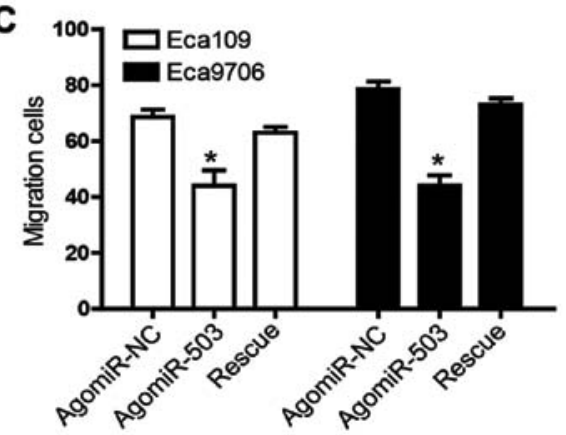

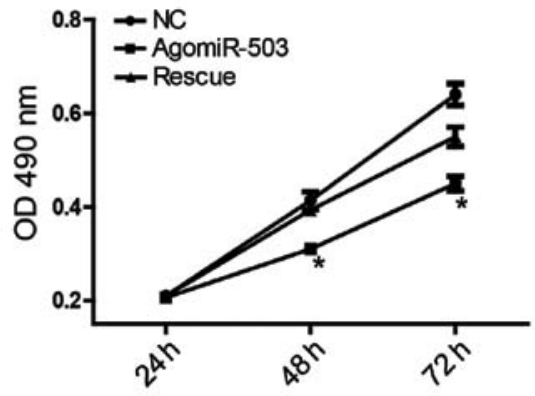

Eca9706

\begin{tabular}{lll}
\hline NC & AgomiR-503 & Rescue
\end{tabular}
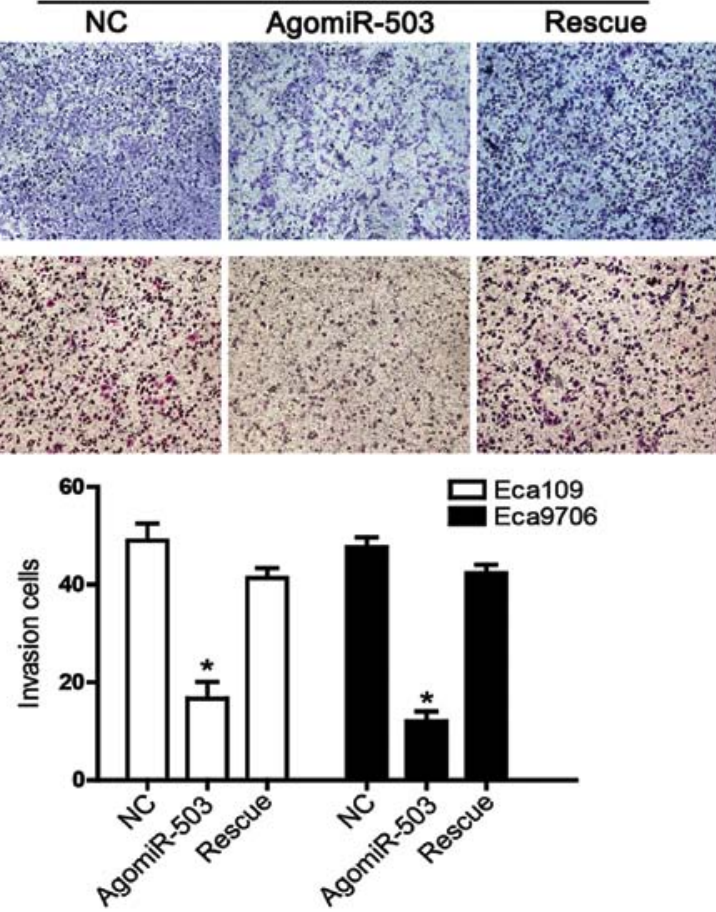

Figure 9. PRKACA restores the aggressive phenotypes of esophageal squamous cell carcinoma (ESCC) which are suppressed by miR-503. (A) A CCK-8 assay revealed that PRKACA 'rescued'/restor4ed the proliferative capacity of ESCC cells following its suppression by miR-503. (B and C) A Transwell assay revealed that PRKACA promoted the migration and invasion of ESCC cells following their suppression by miR-503. "P<0.05; all experiments were repeated 3 times.

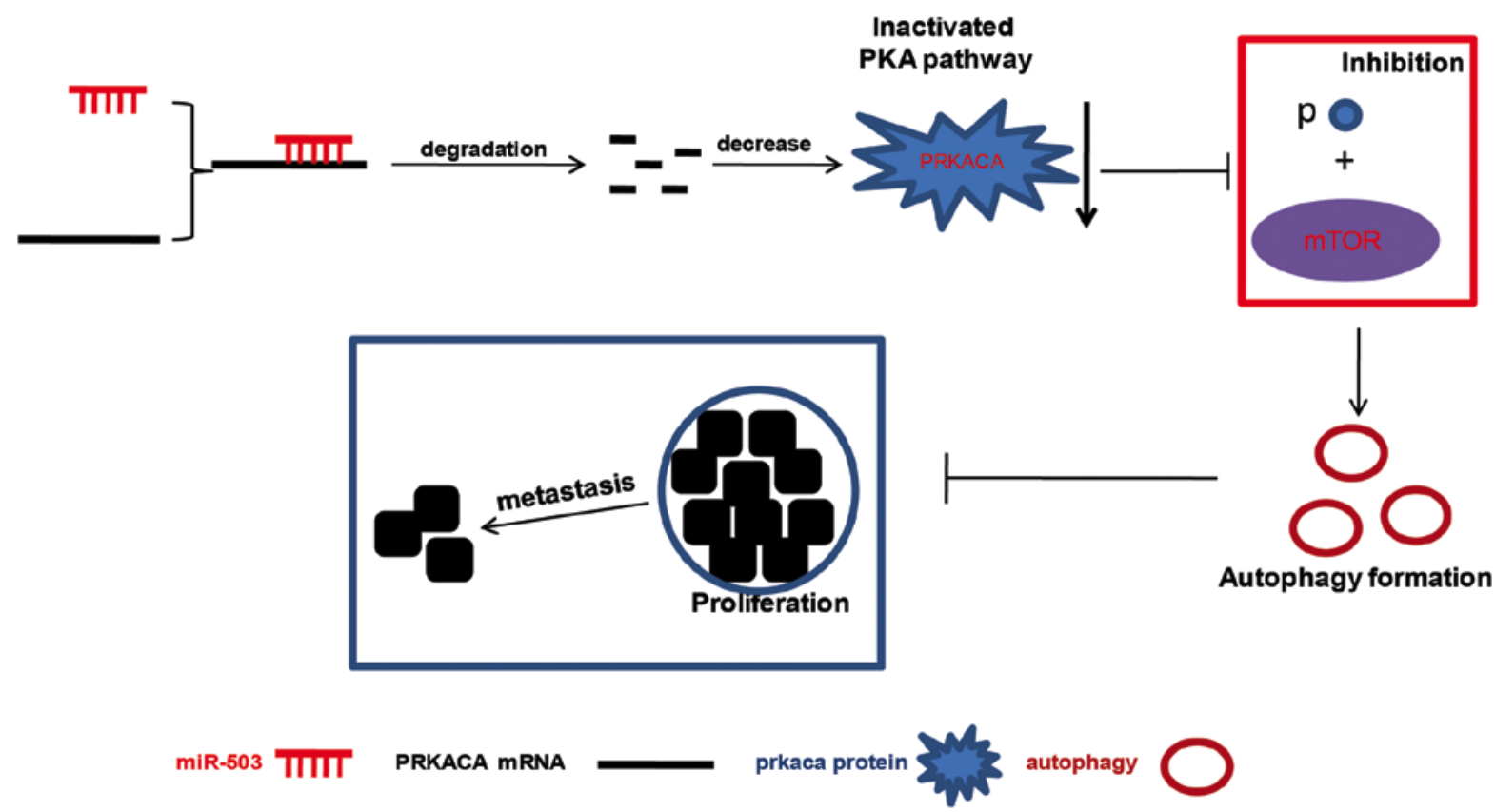

Figure 10. The diagram illustrates the mechanisms of action of miR-503 in esophageal squamous cell carcinoma (ESCC). 
we confirmed that the expression of miR-503 was markedly upregulated in the ESCC tissues when compared with the adjacent non-tumor tissues. It should be noted that not all the patients exhibited a higher miR-503 expression in their tumor tissues. Thus, in the future, we aim to examine its expression in a greater number of patients with ESCC, as in this case, a greater number of patients may be found to have a lower expression of miR-503 in their tumor tissue. Moreover, we also determined the decreased expression of miR-503 in ESCC tissues with lymph node metastasis, at a higher TNM stage (III or IV) or of poor differentiation, rather than in those with no metastasis, a lower TNM stage (I or II) or of high/moderate differentiation. A higher expression of miR-503 was also observed in the ESCC cells. These data thus indicate a suppressive role of miR-503 in the development of ESCC. Indeed, increasing evidence has indicated that miR-503 is involved in tumor metastasis and invasion. For instance, miR-503 has been shown to suppress cell proliferation by targeting L1CAM in osteosarcoma (39); Peng et al confirmed that miR-503 inhibited gastric cancer cell growth and epithelial-to-mesenchymal transition (40). Yang et al found that mature miR-503 suppressed cellular metastasis by targeting PI3K p85 and IKK- $\beta$ in non-small cell lung cancer (34). In this study, we found that the ectopic expression of miR-503 inhibited the proliferation, migration and invasion of ESCC cells in vitro and in vivo. Taken together, our data suggested that miR-503 expression was increased in ESCC tissues, and inhibited the proliferation and metastasis of ESCC cells.

As an evolutionarily conserved biological process, autophagy is involved in the development of multiple tumors, via processes such as cell death and chemosensitivity (41). Intriguingly, accumulating evidence has indicated that miR-503 regulates the chemosensitivity of tumor cells via different target genes, indicating a regulatory role of miR-503 in autophagy. For instance, miR-503 has been shown to regulate the cisplatin resistance of non-small cell lung cancer cells by targeting Bcl-2 (42). In colorectal cancer, miR-503 conferred drug resistance by targeting PUMA (24). In our study, we confirmed that miR-503 triggers the autophagy of ESCC cells by western blot analysis and laser scanning confocal array. Moreover, we observed the formation of autophagosomes in the indicated cells under an electron microscope. Autophagy also affected the development of tumors; thus, we further investigated whether the miR-503-mediated inhibition of ESCC cells was associated with autophagy. Our results revealed that the inhibition of autophagy by 3-MA restored the aggressive phenotype of ESCC cells which was suppressed by miR-503. Taken together, our data indicated that miR-503 inhibited the proliferation and metastasis of ESCC cells by triggering autophagy.

It is well established that miR-503 exerts its function by targeting the specific 3'-UTR of multiple mRNAs in different types of tumor, such as IGF-1R and Bcl-2 (42). However, the target of miR-503 in ESCC remained unclear. In our study, we identified PRKACA as the target of miR-503 in ESCC. Several studies have reported that PRKACA acts as an oncogene in the process of tumor carcinogenesis and progression. For instance, Beristain et al confirmed that the activation of PKA signaling could drive mammary tumorigenesis via Src (43). PRKACA mediated the resistance to HER2-targeted therapy and restored anti-apoptotic signaling in tumor cells in breast cancer (44). Moreover, mutant PRKACA has been reported to promote the development of adrenal adenomas (45). Consistent with these data, we found that PRKACA promoted the proliferation and metastasis of ESCC cells. Moreover, PRKACA overexpression 'rescued' the aggressive phenotype of ESCC cells which was suppressed by miR-503. Furthermore, we found that miR-503 expression negatively correlated with PRKACA in ESCC tissues. These results suggested that miR-503 inhibited the proliferation and metastasis of ESCC cells by targeting PRKACA.

To further explore the underlying mechanisms of PRKACA in autophagy regulation, we assessed the literature and found that the PKA pathway has been implicated in the activity of the mTOR pathway (46). In the current study, western blot analysis revealed that the expression of phosphorylated mTOR protein was markedly attenuated in the indicated cells with PRKACA silencing, or increased in the cells with PRKACA upregulation. It is well established that mTOR can negatively regulate autophagy $(47,48)$; our data indicated that miR-503 regulated autophagy by inhibiting PKA/mTOR signaling. Convincing evidence has indicated that autophagy not only functions as a tumor suppressor, but also generates ATP and other essential biochemical molecules necessary for tumor cell survival under adverse conditions. Lan et al stated that autophagy inhibited hepatocellular carcinoma proliferation, EMT, metastasis and invasion by degrading the oncogene miR-224 (49). Grassi et al demonstrated that autophagy suppressed the epithelial-to-mesenchymal transition of hepatocytes by promoting Snail degradation (50). Catalano et al demonstrated that autophagy impaired migration and invasion by reversing EMT in glioblastoma cells (51). Our study also found that miR-503-mediated autophagy inhibited the development of ESCC, suggesting that miR-503 suppressed the metastatic potential of ESCC by promoting autophagy.

In conclusion, the present study highlighted the regulatory mechanisms through which miR-503 suppresses the proliferation and metastasis of ESCC cells, and indicated that miR-503 may prove to be a therapeutic target for patients with ESCC.

\section{Acknowledgements}

The authors would like to thank Mr. Xiaoping Tang (Experiment Medicine Center, The Affiliated Hospital of Southwest Medical University) and Mr. Kaiming He (Department of Thoracic Surgery, The Affiliated Hospital of Southwest Medical University) for providing valuable technical support and writing assistance.

\section{Funding}

This study was supported by two Joint Fund of Technology Department, Sichuan Province (nos. 2014TSX-0102 and 2015SX-00010), and by the Youth Foundation of the Affiliated Hospital of South West Medical University (16025), and by Sichuan Science and Technology Plan projects (no. 2016RZ0076) and Health and Family Planning Commission of Sichuan Province Research Program (no. 16ZD033).

\section{Availability of data and materials}

The analyzed datasets generated during the study are available from the corresponding author on reasonable request. 


\section{Authors' contributions}

DR and TD conceived and designed the study. JW and FG performed the experiments and wrote the manuscript. TX, CW, $\mathrm{ZH}$ and XY collected the samples. XD, YL, XH and GL were involved in the animal experiments and edited the manuscript. All authors have read and approved the final manuscript.

\section{Ethics approval and consent to participate}

All tissue samples from ESCC patients, who underwent complete surgical resection at the Department of Thoracic Surgery of the Affiliated Hospital of Southwest Medical University (Luzhou, China), were collected after obtaining written informed consent. This study was approved by the Ethics Review Board at Southwest Medical University, Luzhou, China. For experiments involving animals, all experimental procedures were approved by the Institutional Animal Care and Use Committee of Southwest Medical University.

\section{Consent for publication}

Not applicable.

\section{Competing interests}

The authors declare that they have no competing interests.

\section{References}

1. He C, Wu S, Gao A, Su Y, Min H, Shang ZF, Wu J, Yang L, Ding WQ and Zhou J: Phosphorylation of ETS-1 is a critical event in DNA polymerase iota-induced invasion and metastasis of esophageal squamous cell carcinoma. Cancer Sci 108: 2503-2510, 2017

2. Chen XX, Zhong Q, Liu Y, Yan SM, Chen ZH, Jin SZ, Xia TL, Li RY, Zhou AJ, Su Z, et al: Genomic comparison of esophageal squamous cell carcinoma and its precursor lesions by multiregion whole-exome sequencing. Nat Commun 8: 524, 2017.

3. Hou Z, Abudureheman A, Wang L, Hasim A, Ainiwaer J, Zhang H, Niyaz M, Upur H and Sheyhidin I: Expression, prognosis and functional role of Thsd7a in esophageal squamous cell carcinoma of Kazakh patients, Xinjiang. Oncotarget 8: 60539-60557, 2017.

4. Qi Y, Cao KX, Xing FC, Zhang CY, Huang Q, Wu K, Wen FB Zhao $\mathrm{S}$ and Li X: High expression of MAGE-A9 is associated with unfavorable survival in esophageal squamous cell carcinoma. Oncol Lett 14: 3415-3420, 2017.

5. Chan CM, Lai KKY, Ng EKO, Kiang MN, Kwok TWH, Wang HK, Chan KW, Law TT, Tong DK, Chan KT, et al: Serum microRNA$193 \mathrm{~b}$ as a promising biomarker for prediction of chemoradiation sensitivity in esophageal squamous cell carcinoma patients. Oncol Lett 15: 3273-3280, 2018

6. Shang M, Wang X, Zhang Y, Gao Z, Wang T and Liu R: LincRNA-ROR promotes metastasis and invasion of esophageal squamous cell carcinoma by regulating miR-145/FSCN1. Onco Targets Ther 11: 639-649, 2018.

7. Deng XS, Meng X, Venardos N, Song R, Yamanaka K, Fullerton D and Jaggers J: Autophagy negatively regulates proosteogenic activity in human aortic valve interstitial cells. J Surg Res 218: 285-291, 2017.

8. Gu Y, Gao L, Chen Y, Xu Z, Yu K, Zhang D, Zhang G and Zhang X: Sanggenon $C$ protects against cardiomyocyte hypoxia injury by increasing autophagy. Mol Med Rep 16: 8130-8136, 2017.

9. Yu H, Qiu Y, Pang X, Li J, Wu S, Yin S, Han L, Zhang Y, Jin C, Gao X, et al: Lycorine promotes autophagy and apoptosis via TCRP1/Akt/mTOR axis inactivation in human hepatocellular carcinoma. Mol Cancer Ther 16: 2711-2723, 2017.

10. Chen LM, Song TJ, Xiao JH, Huang ZH, Li Y and Lin TY: Tripchlorolide induces autophagy in lung cancer cells by inhibiting the PI3K/AKT/mTOR pathway and improves cisplatin sensitivity in A549/DDP cells. Oncotarget 8: 63911-63922, 2017.
11. Jin C, Zhang S, Yuan J, Wang X, Jin N and Huang J: Hypoxia mediates the decreased sensibility of human lung adenocarcinoma cell H1299 to cisplatin through inducing autophagy. Minerva Chir 73: 113-116, 2017.

12. Ma Z, Chen C, Tang P, Zhang H, Yue J and Yu Z: BNIP3 induces apoptosis and protective autophagy under hypoxia in esophageal squamous cell carcinoma cell lines: BNIP3 regulates cell death. Dis Esophagus 30: 1-8, 2017.

13. Zhou H, Zheng L, Lu K, Gao Y, Guo L, Xu W and Wang X: Downregulation of cohesin loading factor nipped-B-like protein (NIPBL) induces cell cycle arrest, apoptosis, and autophagy of breast cancer cell lines. Med Sci Monit 23: 4817-4825, 2017.

14. Samaras P, Tusup M, Nguyen-Kim TDL, Seifert B, Bachmann H, von Moos R, Knuth A and Pascolo S: Phase I study of a chloroquine-gemcitabine combination in patients with metastatic or unresectable pancreatic cancer. Cancer Chemother Pharmacol 80: 1005-1012, 2017.

15. Zhu J, Zheng Y, Zhang H, Zhu J and Sun H: Low concentration of chloroquine enhanced efficacy of cisplatin in the treatment of human ovarian cancer dependent on autophagy. Am J Transl Res 9: 4046-4058, 2017.

16. Chen HI, Tsai HP, Chen YT, Tsao SC and Chai CY: Autophagy and apoptosis play opposing roles in overall survival of esophageal squamous cell carcinoma. Pathol Oncol Res 22: 699-705, 2016.

17. Cilek EE, Ozturk H and Gur Dedeoglu B: Construction of miRNA-miRNA networks revealing the complexity of miRNAmediated mechanisms in trastuzumab treated breast cancer cell lines. PLoS One 12: e0185558, 2017.

18. Shivapurkar N, Vietsch EE, Carney E, Isaacs C and Wellstein A: Circulating microRNAs in patients with hormone receptorpositive, metastatic breast cancer treated with dovitinib. Clin Transl Med 6: 37, 2017.

19. Hu X, Wang Y, Liang H, Fan Q, Zhu R, Cui J, Zhang W, Zen K, Zhang CY, Hou D, et al: miR-23a/b promote tumor growth and suppress apoptosis by targeting PDCD4 in gastric cancer. Cell Death Dis 8: e3059, 2017.

20. Mei LL, Wang WJ, Qiu YT, Xie XF, Bai J and Shi ZZ: miR-125b-5p functions as a tumor suppressor gene partially by regulating HMGA2 in esophageal squamous cell carcinoma. PLoS One 12: e0185636, 2017.

21. Mei LL, Wang WJ, Qiu YT, Xie XF, Bai J and Shi ZZ: miR-145-5p suppresses tumor cell migration, invasion and epithelial to mesenchymal transition by regulating the $\mathrm{Sp} 1 / \mathrm{NF}-\kappa \mathrm{B}$ signaling pathway in esophageal squamous cell carcinoma. Int J Mol Sci 18: E1833, 2017.

22. Ma T,Zhao Y,Lu Q,Lu Y,Liu Z,Xue T and Shao Y:MicroRNA-30c functions as a tumor suppressor via targeting SNAI1 in esophageal squamous cell carcinoma. Biomed Pharmacother 98: 680-686, 2018.

23. Sun Y, Li L, Xing S, Pan Y, Shi Y, Zhang L and Shen Q: miR-503-3p induces apoptosis of lung cancer cells by regulating p21 and CDK4 expression. Cancer Biomark 20: 597-608, 2017.

24. Xu K, Chen G, Qiu Y, Yuan Z, Li H, Yuan X, Sun J, Xu J, Liang X and Yin P: miR-503-5p confers drug resistance by targeting PUMA in colorectal carcinoma. Oncotarget 8: 21719-21732, 2017.

25. Livak KJ and Schmittgen TD: Analysis of relative gene expression data using real-time quantitative PCR and the $2(-\Delta \Delta C(T))$ method. Methods 25: 402-408, 2001.

26. Qiu T, Zhou L, Wang T, Xu J, Wang J, Chen W, Zhou X, Huang Z, Zhu W, Shu Y, et al: miR-503 regulates the resistance of non-small cell lung cancer cells to cisplatin by targeting Bcl-2. Int J Mol Med 32: 593-598, 2013.

27. Hou S, Tian T, Qi D, Sun K, Yuan Q, Wang Z, Qin Z, Wu Z, Chen Z and Zhang J: S100A4 promotes lung tumor development through $\beta$-catenin pathway-mediated autophagy inhibition. Cell Death Dis 9: 277, 2018.

28. Endo S, Hoshi M, Matsunaga T, Inoue T, Ichihara K and Ikari A: Autophagy inhibition enhances anticancer efficacy of artepillin $C$, a cinnamic acid derivative in Brazilian green propolis. Biochem Biophys Res Commun: Feb 13, 2018 (Epub ahead of print). doi: 10.1016/j.bbrc.2018.02.105.

29. Kastenhuber ER, Lalazar G, Houlihan SL, Tschaharganeh DF, Baslan T, Chen CC, Requena D, Tian S, Bosbach B, Wilkinson JE, et al: DNAJB1-PRKACA fusion kinase interacts with $\beta$-catenin and the liver regenerative response to drive fibrolamellar hepatocellular carcinoma. Proc Natl Acad Sci USA 114: 13076-13084, 2017. 
30. Berthon AS, Szarek E and Stratakis CA: PRKACA: The catalytic subunit of protein kinase A and adrenocortical tumors. Front Cell Dev Biol 3: 26, 2015.

31. Wu D, Cao G, Huang Z, Jin K, Hu H, Yu J and Zeng Y: Decreased miR-503 expression in gastric cancer is inversely correlated with serum carcinoembryonic antigen and acts as a potential prognostic and diagnostic biomarker. Onco Targets Ther 10: 129-135, 2016.

32. Yang X, Zang J, Pan X, Yin J, Xiang Q, Yu J, Gan R and Lei X: miR-503 inhibits proliferation making human hepatocellular carcinoma cells susceptible to 5 fluorouracil by targeting EIF4E. Oncol Rep 37: 563-570, 2017.

33. Lu YC, Chen YJ, Wang HM, Tsai CY, Chen WH, Huang YC, Fan KH, Tsai CN, Huang SF, Kang CJ, et al: Oncogenic function and early detection potential of miRNA-10b in oral cancer as identified by microRNA profiling. Cancer Prev Res (Phila) 5: 665-674, 2012.

34. Yang Y, Liu L, Zhang Y, Guan H, Wu J, Zhu X, Yuan J and Li M: MiR-503 targets PI3K p85 and IKK- $\beta$ and suppresses progression of non-small cell lung cancer. Int J Cancer 135: 1531-1542, 2014.

35. Xu YY,Wu HJ,Ma HD, Xu LP,Huo Y and Yin LR: MicroRNA-503 suppresses proliferation and cell-cycle progression of endometrioid endometrial cancer by negatively regulating cyclin D1. FEBS J 280: 3768-3779, 2013

36. Tömböl Z, Szabó PM, Molnár V, Wiener Z, Tölgyesi G, Horányi J, Riesz P, Reismann P, Patócs A, Likó I, et al: Integrative molecular bioinformatics study of human adrenocortical tumors: microRNA, tissue-specific target prediction, and pathway analysis. Endocr Relat Cancer 16: 895-906, 2009.

37. Corbetta S, Vaira V, Guarnieri V, Scillitani A, Eller-Vainicher C, Ferrero S, Vicentini L, Chiodini I, Bisceglia M, Beck-Peccoz P, et al: Differential expression of microRNAs in human parathyroid carcinomas compared with normal parathyroid tissue. Endocr Relat Cancer 17: 135-146, 2010.

38. Zhao JJ, Yang J, Lin J, Yao N, Zhu Y, Zheng J, Xu J, Cheng JQ, Lin JY and Ma X: Identification of miRNAs associated with tumorigenesis of retinoblastoma by miRNA microarray analysis. Childs Nerv Syst 25: 13-20, 2009.

39. Chong Y, Zhang J, Guo X, Li G, Zhang S, Li C, Jiao Z and Shao M: MicroRNA-503 acts as a tumor suppressor in osteosarcoma by targeting L1CAM. PLoS One 9: e114585, 2014.

40. Peng Y, Liu YM, Li LC, Wang LL and Wu XL: microRNA-503 inhibits gastric cancer cell growth and epithelial-to-mesenchymal transition. Oncol Lett 7: 1233-1238, 2014.

41. Furukawa Y, Takasu A and Yura Y: Role of autophagy in oncolytic herpes simplex virus type 1-induced cell death in squamous cell carcinoma cells. Cancer Gene Ther 24: 393-400, 2017.
42. Wang T, Ge G, Ding Y, Zhou X, Huang Z, Zhu W, Shu Y and Liu P: MiR-503 regulates cisplatin resistance of human gastric cancer cell lines by targeting IGF1R and BCL2. Chin Med J (Engl) 127: 2357-2362, 2014.

43. Beristain AG, Molyneux SD, Joshi PA, Pomroy NC, Di Grappa MA, Chang MC, Kirschner LS, Privé GG, Pujana MA and Khokha R: PKA signaling drives mammary tumorigenesis through Src. Oncogene 34: 1160-1173, 2015.

44. Moody SE, Schinzel AC, Singh S, Izzo F, Strickland MR, Luo L, Thomas SR, Boehm JS, Kim SY, Wang ZC, et al: PRKACA mediates resistance to HER2-targeted therapy in breast cancer cells and restores anti-apoptotic signaling. Oncogene 34: 2061-2071, 2015.

45. Angelousi A, Szarek E, Shram V, Kebebew E, Quezado M and Stratakis CA: Lipofuscin Accumulation in Cortisol-Producing Adenomas With and Without PRKACA Mutations. Horm Metab Res 49: 786-792, 2017.

46. Zhang B, Guo F, Ma Y, Song Y, Lin R, Shen FY, Jin GZ, Li Y and Liu ZQ: Activation of D1R/PKA/mTOR signaling cascade in medial prefrontal cortex underlying the antidepressant effects of 1-SPD. Sci Rep 7: 3809, 2017.

47. de Joussineau C, Sahut-Barnola I, Tissier F, Dumontet T, Drelon C, Batisse-Lignier M, Tauveron I, Pointud JC, LefrançoisMartinez AM, Stratakis CA, et al: mTOR pathway is activated by PKA in adrenocortical cells and participates in vivo to apoptosis resistance in primary pigmented nodular adrenocortical disease (PPNAD). Hum Mol Genet 23: 5418-5428, 2014.

48. Hu J, Cui W, Ding W, Gu Y, Wang Z and Fan W: Globular adiponectin attenuated $\mathrm{H}_{2} \mathrm{O}_{2}$-induced apoptosis in rat chondrocytes by inducing autophagy through the AMPK/mTOR pathway. Cell Physiol Biochem 43: 367-382, 2017.

49. Lan SH, Wu SY, Zuchini R, Lin XZ, Su IJ, Tsai TF, Lin YJ, Wu CT and Liu HS: Autophagy-preferential degradation of MIR224 participates in hepatocellular carcinoma tumorigenesis. Autophagy 10: 1687-1689, 2014.

50. Grassi G, Di Caprio G, Santangelo L, Fimia GM, Cozzolino AM, Komatsu M, Ippolito G, Tripodi M and Alonzi T: Autophagy regulates hepatocyte identity and epithelial-to-mesenchymal and mesenchymal-to-epithelial transitions promoting Snail degradation. Cell Death Dis 6: e1880, 2015.

51. Catalano M, D'Alessandro G, Lepore F, Corazzari M, Caldarola S, Valacca C, Faienza F, Esposito V, Limatola C, Cecconi F, et al: Autophagy induction impairs migration and invasion by reversing EMT in glioblastoma cells. Mol Oncol 9: 1612-1625, 2015.

This work is licensed under a Creative Commons Attribution-NonCommercial-NoDerivatives 4.0 International (CC BY-NC-ND 4.0) License. 